\title{
A new approach to modelling micro-explosions in composite droplets
}

\author{
Sergei S. Sazhin ${ }^{1, *}$, Tali Bar-Kohany ${ }^{2,3}$, Zuhaib Nissar ${ }^{4}$, Dmitrii Antonov ${ }^{5}$, \\ Pavel A. Strizhak ${ }^{5}$, Oyuna D. Rybdylova ${ }^{1}$ \\ ${ }^{1}$ Advanced Engineering Centre, School of Computing, Engineering and Mathematics, \\ University of Brighton, Brighton, BN2 4GJ, UK \\ ${ }^{2}$ School of Mechanical Engineering, Tel Aviv University, Tel Aviv, Israel \\ ${ }^{3}$ Mechanical Engineering Department, nrcn, Israel \\ ${ }^{4}$ Center for Automotive Research and Electric Mobility (CAREM), Universiti Teknologi \\ PETRONAS, 32610 - Seri Iskandar, Perak, Malaysia \\ ${ }^{5}$ National Research Tomsk Polytechnic University, Lenin Avenue, Tomsk 634050, Russia \\ July 6, 2020
}

\begin{abstract}
A new approach to modelling puffing and micro-explosion in composite water/fuel droplets is proposed. This approach is based on the assumption previously made that a spherical water sub-droplet is located in the centre of a spherical fuel (n-dodecane) droplet. The heating of a fuel droplet is described by the heat conduction equation with the Robin boundary condition at its surface and continuity conditions at the fuel-water interface. The analytical solution to this equation, obtained at each time step, is incorporated into the numerical code and used for the analysis of droplet heating and evaporation. The effects of droplet thermal swelling are taken into account. The results of calculations using this code allowed us to obtain the time evolution of the temperature at the water/fuel interface and the evolution of time derivative of this temperature $(\dot{T})$ with time in the same location. Using the original and previously published experimental data, two new correlations for the nucleation temperatures $T_{N}$ as functions of $\dot{T}$, valid in the range $0 \leq \dot{T} \leq 10^{6}$ $\mathrm{K} / \mathrm{s}$, are suggested. Using these correlations and the values of $\dot{T}$ inferred from
\end{abstract}

${ }^{*}$ Corresponding author. E-mail S.Sazhin@brighton.ac.uk 
the analysis, the time evolutions of the nucleation temperatures $T_{N}$ at the water-fuel interfaces are obtained. The predicted values of $T_{N}$ are compared with the values of temperature at this interface $T_{w}$. The time instant when $T_{w}=T_{N}$ is associated with the time instant when puffing/micro-explosion starts.

Keywords: Composite water/fuel droplets, micro-explosions, droplet heating/evaporation, Robin boundary conditions, nucleation temperature.

\section{Nomenclature}

$\begin{array}{ll}B_{M(T)} & \text { Spalding mass }(\text { heat }) \text { transfer number }[-] \\ c & \text { specific heat capacity }[\mathrm{J} /(\mathrm{kg} \mathrm{K})] \\ D & \text { diffusion coefficient }\left[\mathrm{m}^{2} / \mathrm{s}\right] \\ h & \text { convective heat transfer coefficient }\left[\mathrm{W} /\left(\mathrm{m}^{2} \mathrm{~K}\right)\right] \\ k & \text { thermal conductivity }[\mathrm{W} /(\mathrm{m} \mathrm{K})] \\ L & \text { heat of evaporation }[\mathrm{J} /(\mathrm{kg})] \\ \mathrm{Le} & \text { Lewis number }[-] \\ \dot{m}_{d} & \text { evaporation rate }[\mathrm{kg} / \mathrm{s}] \\ \mathrm{Nu} & \text { Nusselt number }[-] \\ p & \text { pressure }[\mathrm{kPa}] \\ P & \text { radiation source term }[\mathrm{K} / \mathrm{s}] \\ \dot{q} & \text { heat transferred to the droplet }[\mathrm{W}] \\ R & \text { distance from the droplet centre }[\mathrm{m}] \\ t & \text { time }[\mathrm{s}] \\ T & \text { temperature }[\mathrm{K}] \\ v_{n} & \text { eigenfunctions }[-] \\ V_{w} & \text { volume fraction of water }[-] \\ Y & \text { mass fraction }[-]\end{array}$

\section{Greek symbols}

\section{$\Delta t$}

$\kappa$

$\lambda_{n}$

$\xi$

$\rho$ time step $[\mathrm{s}]$

thermal diffusivity $\left[\mathrm{m}^{2} / \mathrm{s}\right]$

eigenvalues $[\mathrm{m} / \sqrt{\mathrm{s}}]$

$\frac{R}{R_{d}}[-]$

density $\left[\mathrm{kg} / \mathrm{m}^{3}\right]$

\section{Subscripts}




$\begin{array}{ll}\text { avg } & \text { average } \\ B & \text { boiling } \\ d & \text { droplet } \\ (e) & \text { evaporation } \\ \text { eff } & \text { effective } \\ f & \text { fuel (n-dodecane) } \\ g & \text { ambient gas } \\ l & \text { liquid } \\ N & \text { nucleation } \\ p & \text { at constant pressure } \\ s & \text { surface } \\ (s) & \text { thermal swelling } \\ (t) & \text { thermal swelling and evaporation } \\ v & \text { fuel vapour } \\ w & \text { water or water/fuel interface } \\ 0 & \text { initial conditions } \\ 1 & \text { end of the time step } \\ \infty & \text { ambient conditions }\end{array}$

\section{Introduction}

As has been widely discussed in the literature, and in our recent paper [1], puffing/micro-explosion in water/fuel composite droplets leads to their rapid disintegration and can produce a substantial improvement in internal combustion engine performance. Puffing is the partial ejection of some of the water dispersed within a fuel droplet outside that droplet, while the term micro-explosion refers to the complete break-up of the droplet.

The practical importance of puffing/micro-explosion was discussed in our previous papers $[1,2]$. To summarise the discussion presented in these papers, we mention that these processes can lead to the reduction of exhaust emissions while improving engine performance, without requiring any engine modification. The presence of water in fuel can result in longer ignition delays due to the lower volatility and higher viscosity of water [3]. The reduction in $\mathrm{NO}_{\mathrm{x}}$ emissions can be linked with the reduction in in-cylinder temperature due to the evaporation of water [4].

The results of experimental studies of this phenomenon and various approaches to its modelling are presented in numerous papers, some of which 
are discussed in $[1,2]$.

The experiments described in [1] are focused on relatively small droplets with diameters less than $250 \mu \mathrm{m}$. These experiments were performed in a vessel with air at temperature $700 \mathrm{~K}$ and at atmospheric pressure. The time to puffing/micro-explosion was recorded with the help of a high-speed camera fitted with a long-distance microscope. The microscope images were analysed to measure the droplet initial diameters and the time to puffing/microexplosion. In the experiments a resolution of $256 \times 800$ pixels $^{2}, 12,000$ frames per second, and exposure time of $2 \mu$ s were used. The uncertainty for the time to puffing/micro-explosion was related to the identification of the frames for droplet creation and start of puffing/micro-explosion, which corresponded to $\pm 167 \mu \mathrm{s}$. The uncertainty for the droplet diameter was attributed to the detection of the droplet perimeter; this was estimated as $\pm 37 \mu \mathrm{m}$. Droplets with diameters smaller than $50 \mu \mathrm{m}$ were ignored due to the experimental uncertainty. The time to puffing/micro-explosion was shown to increase with increasing droplet initial diameters.

In contrast to [1], the experiments described in [2] are focused on much larger droplets with diameters in the range 1-3 $\mathrm{mm}$. Micro-explosions were demonstrated to be the key mechanism of disintegration of composite fuel/water droplets in air at temperatures $850-1050^{\circ} \mathrm{C}$. It was shown that the maximal number of child droplets, generated during the disintegration process, was observed when the diameters of these droplets were close to $0.2 \mathrm{~mm}$. The time to puffing/micro-explosion for disintegrating parent droplets was shown to stay almost the same when droplet initial diameters increased.

A typical development of the puffing/micro-explosion phenomenon is illustrated in Figure 1. The first frame in this figure shows the state of the droplet just before puffing/micro-explosion starts (only heating, evaporation and swelling processes take place but these are not visible in the figure). The second frame shows the start of the puffing/micro-explosion when individual child droplets can be clearly seen. In the third frame, the fully developed micro-explosion is shown (a cloud of small child droplets is visible as the black spot in the middle of the figure).

Perhaps the most advanced models of puffing/micro-explosion are described in $[7,8,9,5]$. Although the models described in these papers are crucial for understanding the physics of the processes leading to puffing and micro-explosions in droplets, their applicability for solving practical engineering problems is open to question. Among simplified models of the process, we can mention those suggested in $[10,1,11]$. 


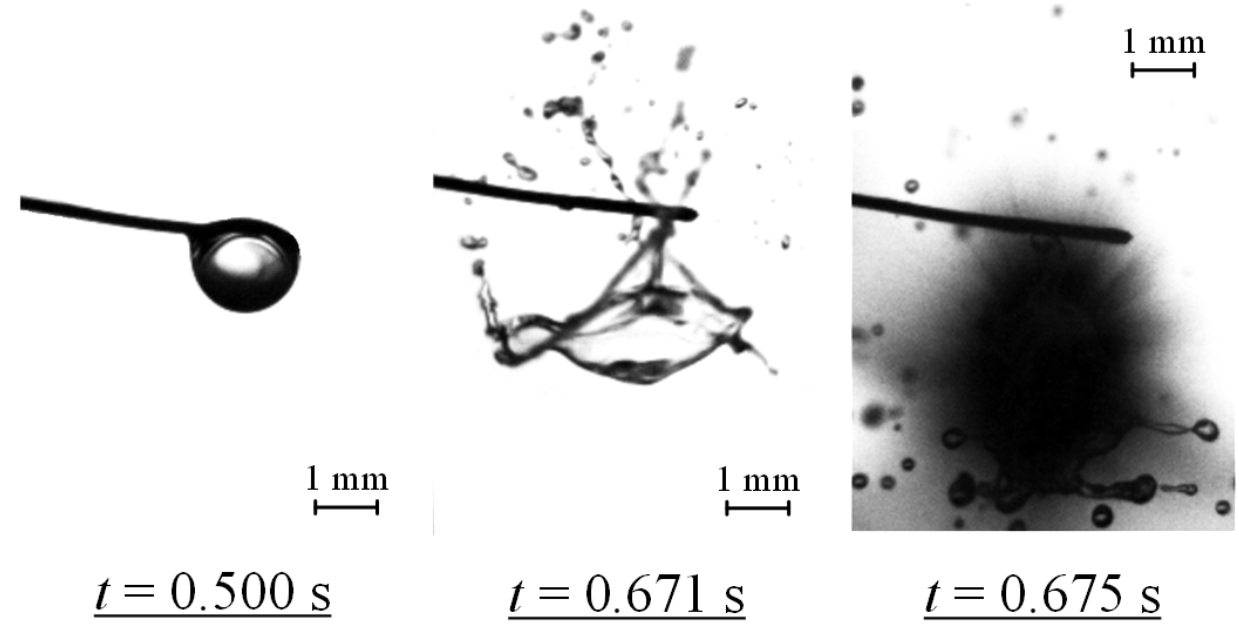

Figure 1: Typical dynamics of puffing/micro-explosion in the experiments performed at National Research Tomsk Polytechnic University [5]. A Diesel fuel droplet with initial radius $R_{d 0}=0.75 \mathrm{~mm}$ and volume fraction of water $V_{w 0}=10 \%$ was placed in an air flow with atmospheric pressure, temperature $T_{g}=723 \mathrm{~K}$ and constant velocity $U_{g}=3 \mathrm{~m} / \mathrm{s}$. $t=0 \mathrm{~s}$ refers to the time instant when the droplet was placed in the air flow.

The model suggested by Girin [10] focuses on the final stage of the puffing process when water inside fuel droplets has evaporated, and the problem of the development of puffing refocuses on the interaction between water vapour and the liquid fuel shell around this vapour. The stability analysis of the vapour/liquid shell was performed assuming that the fuel shell is initially spherically symmetric. This analysis allowed Girin [10] to predict the number of droplets generated after the shell rupture and their average diameters. These were functions of the initial fuel shell thickness. It was shown that the most likely diameter of the child droplets (generated during rupture) is equal to about a quarter (0.27) of the thickness of the fuel shell before the start of the process. We could not obtain these values from the results of experiments performed in [2]. We could anticipate, however, that this thickness is proportional to the thickness of the fuel shell at the start of the process. In this case, assuming that the volume fraction of water is fixed, based on this model we could anticipate that diameters of the droplets generated during the shell rupture are proportional to the initial fuel droplet radii. Unfortunately, this is not supported by experimental data presented in $[2]$. 
In contrast to [10], the focus of the model described in [1] is on the prediction of the time to puffing/micro-explosion. It is believed that this parameter is particularly important for application in engineering problems. As in [10] it was assumed that a spherical water sub-droplet is placed in the centre of a spherical fuel droplet. The surface temperature of the fuel droplet was fixed; droplet thermal swelling and fuel evaporation were not considered. The time-dependent heat transfer equation was solved analytically inside a composite droplet. The Dirichlet boundary condition at its surface was used. The time instant when the temperature at the water/fuel interface reached the boiling temperature for water was considered to be the beginning of the puffing process leading to micro-explosion. The times to puffing/microexplosion predicted by the model were shown to be consistent with observed times to puffing/micro-explosion for droplets with initial radii less than about $0.5 \mathrm{~mm}$, but not for larger droplets [2].

One of the key limitations of the model suggested in [1] is that it uses the assumption of a fixed droplet surface temperature. This assumption is not consistent with observations [12]. The authors of [11] attempted to relax this assumption and to consider a problem similar to the one considered in [1] but using the Robin rather than Dirichlet boundary conditions for the analytical solution to the heat transfer equation inside a droplet. The Robin boundary conditions are known to describe more adequately than the Dirichlet boundary conditions the process at the surface of fuel droplets [12]. In contrast to [1], the analytical solution obtained in [11] was used not for the whole period of droplet heating but only for a short time step during which the computation was performed (the analytical solution obtained in [11] did not offer a single formula describing droplet heating up to the time instant when puffing/micro-explosion started). As in the case of the model in [1], in the model suggested in [11] the start of puffing/micro-explosion is linked with the time instant when the temperature at the fuel/water interface reaches the boiling temperature of water.

In contrast to [1], in the model described in [11] the effects of evaporation were taken into account but it was assumed that the Lewis number was equal to 1 and the specific heat capacity of gas was the same as that of fuel vapour. The effects of evaporation on the convective heat coefficient were ignored and the Nusselt number was assumed equal to 2. The dependence of thermophysical properties on temperature and the effect of swelling were not taken into account. The analytical solution obtained in [11] and the evaporation model were incorporated into a numerical code. The model developed 
in [11] allowed us to better understand the underlying physics of the processes leading to puffing/micro-explosion. The experimental observations of the micro-explosion delay times for droplets with initial radii less than about $100 \mu \mathrm{m}$ were shown to be compatible with the model predictions. It was shown that puffing/micro-explosions are expected to take place well before the droplet surface temperature becomes equal to the boiling temperature of n-dodecane. This clearly shows the limitation of the model described in [1].

Apart from the above-mentioned limitations of the model described in [11], the analytical solution on which this model was based contained a mistake. This mistake did not affect the conclusions of [11] but it affected some numerical values on the graphs. Also, the models described in [1] and [11] are based on the assumption that puffing, possibly leading to micro-explosion, starts when the temperature at the water/fuel interface reaches the water equilibrium boiling temperature $T_{B}$. At the same time, it is well known that in the transient case when temperature increases quickly enough with time, boiling is expected to take place not at the boiling temperature, but at the nucleation temperature $T_{N}$ which is higher than $T_{B}$. The link between these temperatures has been investigated in many papers, including $[13,14]$.

The aim of this paper is to present the results of the development of a new model for micro-explosions. This model is based on the same assumption that a spherical water sub-droplet is located in the centre of a spherical fuel droplet as used in $[1,11]$. The formulation of the problem for the analytical solution to the heat transfer equation with the Robin boundary condition at the surface of fuel droplet is the same as in the model described in [11], but the resulting analytical solution is different from the one presented in [11]. The effects of evaporation of the fuel droplet are taken into account using the Abramzon and Sirignano model [12] assuming that the droplet is stationary, but without any additional assumptions regarding the values of the Lewis number and the values of the specific heat capacities of fuel vapour and ambient gas. The effect of swelling is taken into account. The effect of replacing the boiling temperature of water with the nucleation temperature, as the temperature at which puffing starts, is investigated.

The analysis of the phase separation process and the formation of a composite state of water in fuel is beyond the scope of the paper. These processes are discussed in many papers, including $[15,16]$.

Basic equations and approximations used in the new model are described in the next section. The results of calculations for parameter values typically used in micro-explosion experiments are presented in Section 3. Section 4 fo- 
cuses on the analysis of the values of the nucleation temperature $T_{N}$ obtained in various experiments at various heating rates $\dot{T}=d T / d t$, and approximations of the dependence of $T_{N}$ on $\dot{T}$. In Section 5 these approximations are applied for the prediction of the time to puffing/micro-explosion within the newly developed model. In the final section, the main results of the paper are summarised.

\section{Basic equations and approximations}

The new model is based on the same assumption regarding the geometry of a composite water/fuel droplet as the models described in $[1,11]$. A stationary water droplet of radius $R_{w}$ is introduced in the centre of a fuel droplet of radius $R_{d}\left(R_{d}>R_{w}\right)$. The evolution of temperature inside the droplet in space and time is described by the following heat transfer equation:

$$
\frac{\partial T}{\partial t}=\kappa\left(\frac{\partial^{2} T}{\partial R^{2}}+\frac{2}{R} \frac{\partial T}{\partial R}\right)+P(t, R)
$$

where

$$
\kappa=\left\{\begin{array}{l}
\kappa_{w}=k_{w} /\left(c_{w} \rho_{w}\right) \quad \text { when } \quad R \leq R_{w} \\
\kappa_{f}=k_{f} /\left(c_{f} \rho_{f}\right) \quad \text { when } \quad R_{w}<R \leq R_{d}
\end{array}\right.
$$

$\kappa_{w(f)}, k_{w(f)}, c_{w(f)}$, and $\rho_{w(f)}$ are the water (liquid fuel) thermal diffusivity, thermal conductivity, specific heat capacity, and density, respectively; $R$ is the distance from the centre of the droplet, $t$ is time. The source term $P(t, R)$ takes into account the volumetric droplet heating (e.g. heating due to thermal radiation).

The model is based on the analytical solution to Equation (1) with the following initial and boundary conditions, including the Robin boundary condition at the droplet surface:

$$
\begin{gathered}
\left.T\right|_{t=0}=\left\{\begin{array}{lll}
T_{w 0}(R) & \text { when } \quad R \leq R_{w} \\
T_{f 0}(R) & \text { when } \quad R_{w}<R \leq R_{d},
\end{array}\right. \\
\left.T\right|_{R=R_{w}^{-}}=\left.T\right|_{R=R_{w}^{+}},\left.\quad k_{w} \frac{\partial T}{\partial R}\right|_{R=R_{w}^{-}}=\left.k_{f} \frac{\partial T}{\partial R}\right|_{R=R_{w}^{+}}, \\
h\left(T_{\text {eff }}-T\left(R_{d}\right)\right)=\left.k_{f} \frac{\partial T}{\partial R}\right|_{R=R_{d}-0},
\end{gathered}
$$


where $h$ is the convection heat transfer coefficient. Consistency between Conditions (3) and (4) is ensured if we assume that $T_{w 0}\left(R_{w}\right)=T_{f 0}\left(R_{w}\right)$.

$$
T_{\text {eff }}=T_{g}+\frac{\rho_{l} L \dot{R}_{d(e)}}{h}
$$

The term $\dot{R}_{d(e)}=d R_{d} / d t$ takes into account the effect of evaporation. It is obtained from the droplet mass evaporation rate described by the following equation [12]:

$$
\dot{m}_{d}=-4 \pi R_{d} D_{v} \rho_{\text {total }} \ln \left(1+B_{M}\right),
$$

where $B_{M}=\left(Y_{v s}-Y_{v \infty}\right) /\left(1-Y_{v s}\right)$ is the Spalding mass transfer number, $Y_{v s}$ $\left(Y_{v \infty}\right)$ is the mass fraction of fuel vapour near the droplet surface (ambient gas), $D_{v}$ is the diffusion coefficient between the vapour and ambient gas (air), the total density of the mixture of fuel vapour and air $\rho_{\text {total }}=\rho_{g}+\rho_{v}$ is assumed not to depend on the distance from the droplet surface.

We present the convection heat transfer coefficient $h$ as $h=k_{g} \mathrm{Nu} /\left(2 R_{d}\right)$, where the Nusselt number for stationary evaporation droplets is presented as:

$$
\begin{gathered}
\mathrm{Nu}=2 \frac{\ln \left(1+B_{T}\right)}{B_{T}}, \\
B_{T}=\frac{c_{p v}\left(T_{g}-T_{s}\right)}{L\left(T_{s}\right)-\left(\left|\dot{q}_{d}\right| / \dot{m}_{d}\right)}
\end{gathered}
$$

is the Spalding heat transfer number ${ }^{1}, T_{g}\left(T_{s}\right)$ is gas (surface) temperature, $L$ is specific heat of evaporation, $c_{p v}$ is specific vapour heat capacity at constant pressure, $\left|\dot{q}_{d}\right|$ is heat spent on raising the droplet temperature.

For stationary droplets, $B_{T}$ and $B_{M}$ are linked by the following relationship [12]:

$$
B_{T}=\left(1+B_{M}\right)^{\varphi}-1
$$

where

$$
\varphi=\left(\frac{c_{p v}}{c_{p g}}\right) \frac{1}{\mathrm{Le}}
$$

Le $=k_{g} /\left(c_{p g} \rho_{\text {total }} D_{v g}\right)$ is the Lewis number.

The first attempt to solve Equation (1) subject to the above-mentioned initial and boundary conditions was made in [11]. Unfortunately, as previously mentioned, there was a mistake in the analytical solution obtained in

\footnotetext{
${ }^{1}$ The effect of evaporation on $\mathrm{Nu}$ was ignored in [11] where it was assumed that $\mathrm{Nu}=2$
} 
[11]. This mistake did not affect the key conclusions made in [11], but affected the numerical values in the plots. The correct expression for the distribution of temperature inside the composite droplets, using the same assumptions as in [11], is the following (the details are presented in Appendix 1):

$$
T=\frac{1}{R_{d} \xi}\left[\sum_{n=1}^{\infty} \Theta_{n}(t) v_{n}(\xi)+\frac{\mu_{0} \xi}{1+h_{0}}\right],
$$

where

$$
\begin{aligned}
& \Theta_{n}(t)=\left(q_{n}+f_{n} \mu_{0}\right) \exp \left(-\frac{\lambda_{n}^{2} t}{R_{d}^{2}}\right)+\frac{p_{n}}{\lambda_{n}^{2}}\left[1-\exp \left(-\frac{\lambda_{n}^{2} t}{R_{d}^{2}}\right)\right] \\
& f_{n}=\frac{1}{\left\|v_{n}\right\|^{2}} \int_{0}^{1} f(\xi) v_{n}(\xi) b \mathrm{~d} \xi \\
& =\frac{1}{\left\|v_{n}\right\|^{2}}\left\{\int_{0}^{\xi_{w}} \frac{-\xi}{1+h_{0}} \frac{\sin \left(\lambda_{n} a_{w} \xi\right)}{\sin \left(\lambda_{n} a_{w} \xi_{w}\right)} k_{w} a_{w}^{2} \mathrm{~d} \xi+\int_{\xi_{w}}^{1} \frac{-\xi}{1+h_{0}} \frac{\sin \left(\lambda_{n} a_{f} \xi+\beta_{n}\right)}{\sin \left(\lambda_{n} a_{f} \xi_{w}+\beta_{n}\right)} k_{f} a_{f}^{2} \mathrm{~d} \xi\right\} \\
& q_{n}=\frac{1}{\left\|v_{n}\right\|^{2}} \int_{0}^{1} F_{0}(\xi) v_{n}(\xi) b \mathrm{~d} \xi \\
& =\frac{1}{\left\|v_{n}\right\|^{2}}\left\{\int_{0}^{\xi_{w}} R_{d} \xi T_{0}\left(R_{d} \xi\right) \frac{\sin \left(\lambda_{n} a_{w} \xi\right)}{\sin \left(\lambda_{n} a_{w} \xi_{w}\right)} k_{w} a_{w}^{2} \mathrm{~d} \xi+\int_{\xi_{w}}^{1} R_{d} \xi T_{0}\left(R_{d} \xi\right) \frac{\sin \left(\lambda_{n} a_{f} \xi+\beta_{n}\right)}{\sin \left(\lambda_{n} a_{f} \xi_{w}+\beta_{n}\right)} k_{f} a_{f}^{2} \mathrm{~d} \xi\right\} \\
& p_{n}=\frac{1}{\left\|v_{n}\right\|^{2}} \int_{0}^{1} R_{d}^{3} \xi \tilde{P}(\xi) v_{n}(\xi) b \mathrm{~d} \xi \\
& =\frac{1}{\left\|v_{n}\right\|^{2}}\left\{\int_{0}^{\xi_{w}} R_{d}^{3} \xi \tilde{P}(\xi) \frac{\sin \left(\lambda_{n} a_{w} \xi\right)}{\sin \left(\lambda_{n} a_{w} \xi_{w}\right)} k_{w} a_{w}^{2} \mathrm{~d} \xi+\int_{\xi_{w}}^{1} R_{d}^{3} \xi \tilde{P}(\xi) \frac{\sin \left(\lambda_{n} a_{f} \xi+\beta_{n}\right)}{\sin \left(\lambda_{n} a_{f} \xi_{w}+\beta_{n}\right)} k_{f} a_{f}^{2} \mathrm{~d} \xi\right\} \\
& f(\xi) \equiv \frac{-\xi}{1+h_{0}}=\sum_{n=1}^{\infty} f_{n}(t) v_{n}(\xi) \\
& F_{0}(\xi) \equiv R_{d} \xi T_{0}\left(R_{d} \xi\right)=\sum_{n=1}^{\infty} q_{n}(t) v_{n}(\xi), \quad R_{d}^{3} \xi \tilde{P}(\xi)=\sum_{n=1}^{\infty} p_{n}(t) v_{n}(\xi) \\
& v_{n}(\xi)= \begin{cases}\frac{\sin \left(\lambda_{n} a_{w} \xi\right)}{\sin \left(\lambda_{n} a_{w} \xi_{w}\right)} & \text { when } 0 \leq \xi \leq \xi_{w} \\
\frac{\sin \left(\lambda_{n} a_{f} \xi+\beta_{n}\right)}{\sin \left(\lambda_{n} a_{f} \xi_{w}+\beta_{n}\right)} & \text { when } \quad \xi_{w}<\xi \leq 1\end{cases}
\end{aligned}
$$


$\beta_{n}$ is $\beta\left(\lambda=\lambda_{n}\right)$,

$$
\begin{gathered}
\left\|v_{n}\right\|^{2}=\int_{0}^{1} v_{n}^{2} b \mathrm{~d} \xi=\frac{\sqrt{c_{w} \rho_{w} k_{w}}}{\lambda_{n} \sin ^{2}\left(\lambda_{n} a_{w} \xi_{w}\right)}\left[\frac{a_{w} \lambda_{n} \xi_{w}}{2}-\frac{\sin \left(2 a_{w} \lambda_{n} \xi_{w}\right)}{4}\right] \\
+\frac{\sqrt{c_{f} \rho_{f} k_{f}}}{\lambda_{n} \sin ^{2}\left(\lambda_{n} a_{f} \xi_{w}+\beta_{n}\right)}\left\{\frac{a_{f} \lambda_{n}\left(1-\xi_{w}\right)}{2}-\frac{\sin \left(2 \lambda_{n} a_{f}+2 \beta_{n}\right)-\sin \left(2 \lambda_{n} a_{f} \xi_{w}+2 \beta_{n}\right)}{4}\right\}, \\
\beta=\cot ^{-1}\left[\frac{k_{f}-k_{w}}{k_{f} a_{f} \xi_{w} \lambda}+\frac{k_{w} a_{w}}{k_{f} a_{f}} \cot \left(a_{w} \lambda \xi_{w}\right)\right]+i \pi-a_{f} \lambda \xi_{w},
\end{gathered}
$$

$i=0,1,2,3, \ldots$., we restrict the analysis to the case when $i=0$ (the values of $v$ would be the same for other values of $i$ ), a countable set of positive eigenvalues $\lambda_{n}$ is obtained from the boundary condition at $\xi=1$ :

$$
\lambda_{n} a_{f} \cos \left(\lambda_{n} a_{f}+\beta\right)+h_{0} \sin \left(\lambda_{n} a_{f}+\beta\right)=0 .
$$

The following new parameters were introduced:

$$
\begin{gathered}
H=\frac{h}{k_{f}}-\frac{1}{R_{d}}, \quad \mu=\frac{R_{d}}{k_{f}}\left(h T_{g}+\rho_{l} L_{f} \dot{R}_{d}\right), \quad \xi=R / R_{d}, \quad \tilde{P}(\xi)=P\left(\xi R_{d}\right), \\
F(t, \xi)=u(t, R) \equiv T(R, t) R, \quad h_{0}=H R_{d}=\frac{h R_{d}}{k_{f}}-1, \\
\mu_{0}=\mu R_{d}=\frac{R_{d}^{2}}{k_{f}}\left(h T_{g}+\rho_{l} L_{f} \dot{R}_{d}\right)=\frac{R_{d}^{2} h T_{\mathrm{eff}}}{k_{f}} .
\end{gathered}
$$

In the limit when thermophysical parameters of water and fuel are the same, Solution (11) reduces to Equation (16) of [17] derived for homogeneous droplets (see Appendix 2 for the details). As shown is [11], in the same limit the results obtained in [11] fully agree with those presented earlier in $[17,18]$.

The effect of thermal swelling on the change of droplet radius during the time step is taken into account based on the requirement of conservation of masses of water and liquid fuel during the heating process. For each time step, this requirement leads to the following equations:

$$
\begin{gathered}
R_{w 1}=R_{w 0}\left(\frac{\rho_{w 0}}{\rho_{w 1}}\right)^{1 / 3} \\
\frac{4}{3} \pi\left(R_{d 0}^{3}-R_{w 0}^{3}\right) \rho_{f 0}=\frac{4}{3} \pi\left(R_{d 1}^{3}-R_{w 1}^{3}\right) \rho_{f 1},
\end{gathered}
$$


where subscripts 0 and ${ }_{1}$ refer to the beginning and end of the time step, respectively.

The combination of Equations (13) and (14) allows us to obtain the following expression for the change of droplet radius due to swelling during each time step:

$$
\Delta R_{d(s)} \equiv R_{d 1}-R_{d 0}=R_{d 0}\left\{\left[\left(1-\frac{R_{w 0}^{3}}{R_{d 0}^{3}}\right) \frac{\rho_{f 0}}{\rho_{f 1}}+\frac{R_{w 0}^{3}}{R_{d 0}^{3}} \frac{\rho_{w 0}}{\rho_{w 1}}\right]^{1 / 3}-1\right\}
$$

where subscript $(s)$ indicates the change due to thermal swelling.

The total change of droplet radius due to swelling and evaporation is estimated as:

$$
\Delta R_{d(t)}=\Delta R_{d(s)}+\Delta R_{d(e)}
$$

where the change in droplet radius due to swelling $\left(\Delta R_{d(s)}\right)$ is inferred from Expression (15), while the change of droplet radius due to evaporation $\left(\Delta R_{d(e)}\right)$ can be obtained from Equation (6) as:

$$
\Delta R_{d(e)}=\left(\frac{\dot{m}_{d}}{4 \pi R_{d 0}^{2} \rho_{f 0}}\right) \Delta t
$$

where $\Delta t-t_{1}-t_{0}$.

\section{Analysis}

Calculations based on the models described in the previous section were performed in parallel using Mathematica 12.1 and Matlab R2020a to eliminate the possibility of numerical errors. Fifty and 100 terms were used in series (11); we observed no difference in the results for these numbers of terms. Time steps $1 \mu \mathrm{s}$ and $0.1 \mathrm{~s}$ were used in calculations for the smallest and largest droplets, respectively; 300 cells along the droplet radius were used to calculate integrals for the parameters in (11). The roots of Equation (12) were found using the bisection method with absolute accuracy of $10^{-16}$.

As in [11], it was assumed that pressure is atmospheric and $T_{g}$ is constant. The effects of fuel vapour on gas transport properties were ignored. In contrast to [11], we took into account the temperature dependencies of thermophysical parameters of water and fuel. The thermophysical parameters of water were taken from [19]. Fuel was approximated by n-dodecane and its properties were taken from [20]. 
The droplet surface temperatures $\left(T_{s}\right)$ and average temperatures of water and liquid fuel $\left(T_{\operatorname{avg}(w)}\right.$ and $\left.T_{\operatorname{avg}(f)}\right)$ were calculated at each time step. The specific heat of evaporation and saturated fuel vapour mass fractions were calculated at $T_{s}$. The thermophysical properties of water and liquid fuel were obtained at $T_{\operatorname{avg}(w)}$ and $T_{\operatorname{avg}(f)}$. The air transport properties were obtained using the $2 / 3$ rule [12]. All these properties were found for the initial temperatures or the temperatures obtained at the end of the previous time step. The same applies to the values of $d R_{d} / d t$ used for estimating $T_{\text {eff }}$, and $\mu$ and $\mu_{0}$ in Expressions (A4) and (A8).

Firstly, the model was applied to the analysis of heating/ evaporation/ puffing/ micro-explosion of the same composite n-dodecane/water droplet as in [11]. The droplet initial temperature and ambient gas temperature were taken as $300 \mathrm{~K}$ and $700 \mathrm{~K}$, respectively. The droplet initial radius was taken as $5 \mu \mathrm{m}$, the ambient pressure was set to $101.325 \mathrm{kPa}$ and the initial volume fraction of water $V_{w 0}$ was taken to be $15 \%$ (these are the same parameters as used for Fig. 3 of [11]). Figure 2 shows the spatial temperature distribution at several time instants using the new model. It can be seen that as the droplet heats up and evaporates, $\xi_{w}$ shifts and tends to merge with the droplet surface. The changes in this parameter are attributed to shrinking of droplets due to evaporation. At time instant $t=0.25 \mathrm{~ms}$, the droplet radius reduced to about $4.65 \mu \mathrm{m}$. The effects of swelling are ignored to be consistent with the approximation used to obtain the results shown in Fig. 3 of [11].

As in the case of the models described in $[1,11]$, the start of the puffing/microexplosion process was identified as the time instant when the temperature at the water/fuel interface reached the boiling temperature of water. Later this assumption will be relaxed.

The time to puffing/micro-explosion for the case shown in Figure 2 is $0.135 \mathrm{~ms}$. This can be compared with the time to puffing/micro-explosion predicted by the previous algorithm but using the correct analytical solution $(0.138 \mathrm{~ms})$ and time reported in [11] $(0.21 \mathrm{~ms})$. These results show that the improvements to the heating/evaporation model lead to relatively small improvements in the accuracy of the prediction of the time to puffing/microexplosion.

Plots similar to those shown in Figure 2, but for bigger droplets used in the experiments performed at Tomsk Polytechnic University are presented in Figures 3 (without the effect of swelling) and 4 (with the effect of swelling taken into account). In these experiments, kerosene (Jet A-1) was used as the 


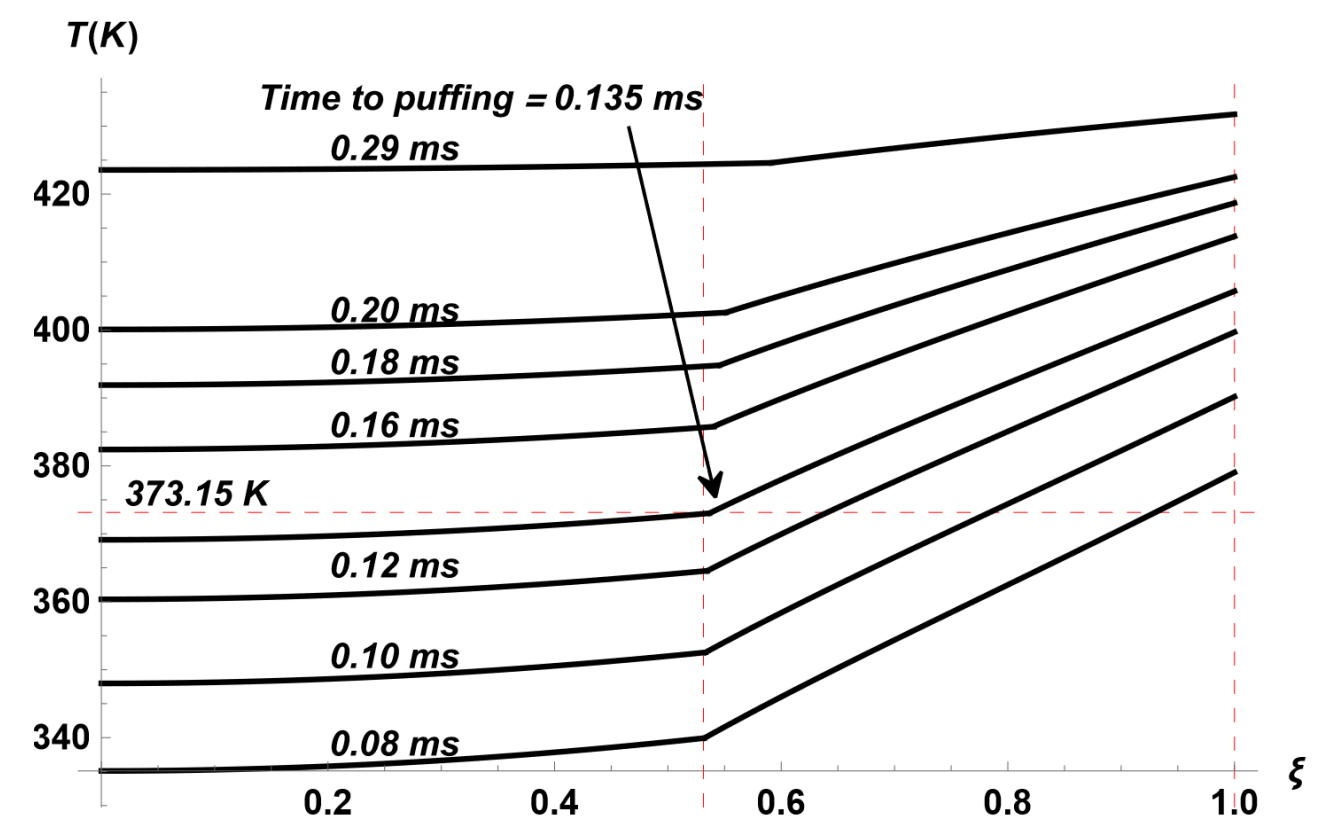

Figure 2: Plots of $T(\xi)$ at several time instants for $R_{d 0}=5 \mu \mathrm{m}, T_{d 0}=300 \mathrm{~K}, T_{g}=700$ $\mathrm{K}, p=1 \mathrm{~atm}=101.325 \mathrm{kPa}, V_{w 0}=15 \%$.

fuel, which was approximated by n-dodecane in the modelling (as in the case shown in Figure 2). A composite water/fuel droplet at initial temperatures $T_{d 0}=300 \pm 3 \mathrm{~K}$ was placed in a hot air. Three values of ambient gas temperature $T_{g}$ were used: $573 \mathrm{~K}, 673 \mathrm{~K}$ and $773 \mathrm{~K}$. The calculations, the results of which are presented in Figures 3 and 4, were performed only for $773 \mathrm{~K}$. The initial droplet radius $R_{d 0}=1.33 \pm 0.03 \mathrm{~mm}$, and initial water volume fraction $V_{w 0}=20 \%$ were used; the experiments were performed at atmospheric pressure.

Comparing Figures 2 and 3 one can see that the evolutions of temperatures inside small and large droplets in space and time are rather similar, but the predicted time to puffing/micro-explosion is much longer for large droplets $(8 \mathrm{~s})$ than for small ones. From a comparison between Figures 3 and 4 it can be seen that taking into account the effect of swelling leads to longer times to puffing $(8.2 \mathrm{~s})$ compared to the case when this effect is ignored (8.0 $\mathrm{s})$. This is attributed to the fact that swelling leads to an increase in the thickness of the fuel shell through which heat is transferred from the surface of the droplet to the water/fuel interface. 


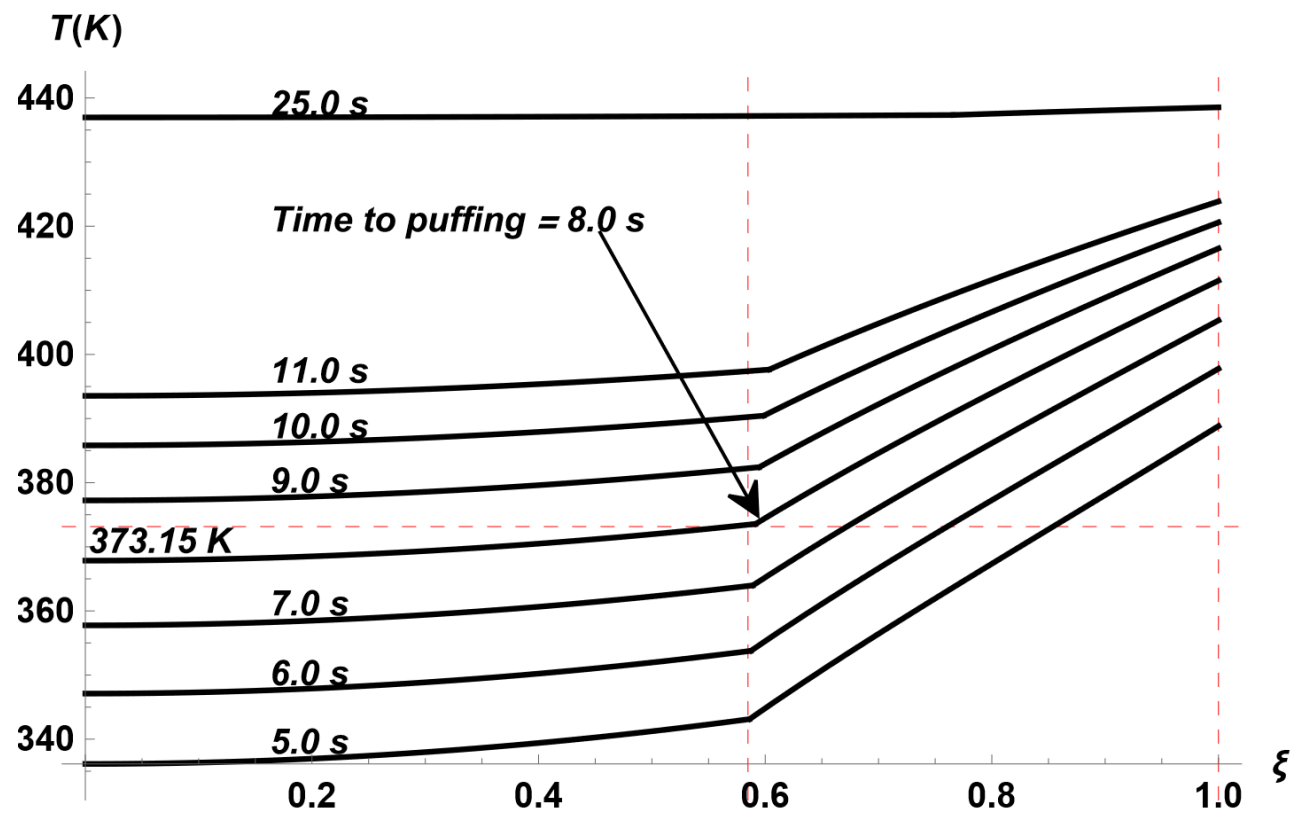

Figure 3: Plots of $T(\xi)$ at several time instants for $R_{d 0}=1.33 \mathrm{~mm}, T_{d 0}=300 \mathrm{~K}, T_{g}=773$ $\mathrm{K}, p=1 \mathrm{~atm}=101.325 \mathrm{kPa}, V_{w 0}=20 \%$. The effects of swelling were not taken into account.

As follows from our analysis, the predicted droplet radius reduces from $1.33 \mathrm{~mm}$ to about $1 \mathrm{~mm}$ over $25 \mathrm{~s}$ when the effects of swelling are ignored. When the effects of swelling are taken into account, this radius reduces to only $1.1 \mathrm{~mm}$ in the same time. This shows that the effect of swelling is worthy of note and should be taken into account in the accurate analysis of the phenomenon.

\section{Boiling versus nucleation temperature}

As mentioned in the Introduction, the models developed in $[1,11]$ are based on the assumption that puffing starts when the temperature at the water/fuel interface reaches the water equilibrium boiling temperature $T_{B}$. The same approach was used in the previous section. At the same time, it is well known that in the transient case when temperature increases with time quickly enough, boiling is expected to take place not at the boiling temperature, but at the nucleation temperature $T_{N}$ which is higher than $T_{B}$. 


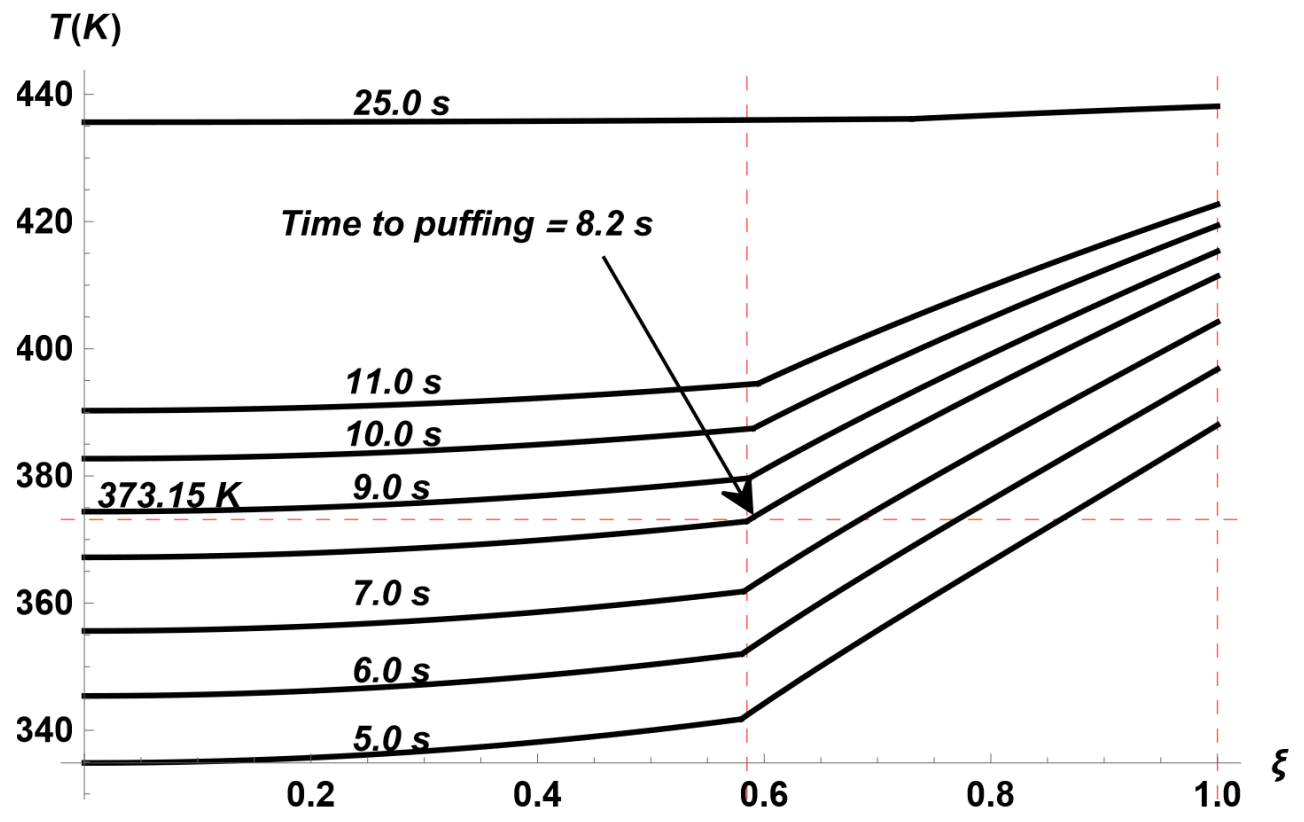

Figure 4: Plots of $T(\xi)$ at several time instants for $R_{d 0}=1.33 \mathrm{~mm}, T_{d 0}=300 \mathrm{~K}, T_{g}=773$ $\mathrm{K}, p=1 \mathrm{~atm}=101.325 \mathrm{kPa}, V_{w 0}=20 \%$. The effects of swelling were taken into account.

This implies that the micro-explosion and puffing events occur while the inner droplet lies within the metastable region (water, in the present study). A metastable system will relax into a stable thermodynamic state sooner or later, depending on the activation sources that prevail [21]. For a homogeneous nucleation, the small fraction of molecules with considerably higher than average energy constitute such an activation source [15]. The scattered nature of the nucleation phenomenon can be seen in Figures 5 and 6. For very high heating rates, the onsets of nucleate boiling (ONB) conditions are less likely to be affected by the surface morphology, as shown in [14]. 'In contrast, heterogeneous nucleation is more likely to be affected by the geometrical characteristics of interfaces. Contaminations, such as colloids, for example, have a much more dominant effect for the low heating rates domain. In the present study we assume that all of the micro-explosion and puffing events are initiated at the interface, and the occurrence of micro-explosions can be understood in a deterministic way. For relatively large droplets with diameters more than $0.5 \mathrm{~mm}$ the validity of the latter assumption was demonstrated in [22]. This is a common assumption made in all previous models of 
the phenomena, including those based on the Direct Numerical Simulation $[7,8,9]$.

The link between $T_{N}$ and $T_{B}$ has been investigated in many papers, including $[13,14]$. The authors of [14] suggested the following correlation for rapid heating rates

$$
T_{N}=T_{B}+0.37 T_{B} \cdot \dot{T}^{10 / J a_{H N}} \quad 10^{5} \leq \dot{T} \leq 10^{9} \mathrm{~K} / \mathrm{s}
$$

where $J a_{H N}=626$ for water.

Using the results presented in $[13,14]$, the following approximate formula, valid for intermediate to high heating rates, was obtained:

$$
T_{N}=385+160 \times \tanh \left(\dot{T} / 10^{5}\right) \quad 10^{2} \leq \dot{T} \leq 10^{6} \mathrm{~K} / \mathrm{s},
$$

All temperatures are in $\mathrm{K}$, and $\dot{T} \equiv d T / d t$ is in $\mathrm{K} / \mathrm{s}$. Both Formulae ((18) and (19)) were obtained at atmospheric pressure.

A comparison between the values of the nucleation temperatures for various $\dot{T}$ inferred from Equations (18) and (19) and those observed experimentally is shown in Figure 5. For this comparison, we used the experimental data previously published in $[23,24,25,26,27,13]$ and those obtained in our original experiments, mentioned earlier. In the latter experiments the two-colour LIP was used to determine the temperatures at the water/fuel interface immediately before and at the time instant when puffing started. The temperature at the time instant when puffing started was identified as the nucleation temperature, while the changes in temperature before this time instant allowed us to obtain the values of $\dot{T}$ used in the above-mentioned correlations. This method is applicable for slow heating rates up to about $250-300 \mathrm{~K} / \mathrm{s}$. The results of the original experiment are shown at $\dot{T}$ close to 100 .

As follows from Figure 5, the agreement between the above-mentioned correlations and experimental data can be considered reasonably good. This allows us to recommend them for practical estimation of nucleation temperatures.

As mentioned earlier, in our original experiments the values of $\dot{T}$ were always below $250-300 \mathrm{~K} / \mathrm{s}$, and on many occasions they were less than 100 $\mathrm{K} / \mathrm{s}$. This led to severe limitations on the applicability of Formula (19) to the practical estimation of $T_{N}$.

This was the motivation behind the development of a new correlation applicable to low heating rates:

$$
T_{N}=T_{B}+12 \times \tanh (\dot{T} / 50) ; \quad 0 \leq \dot{T} \leq 300 \mathrm{~K} / \mathrm{s} .
$$




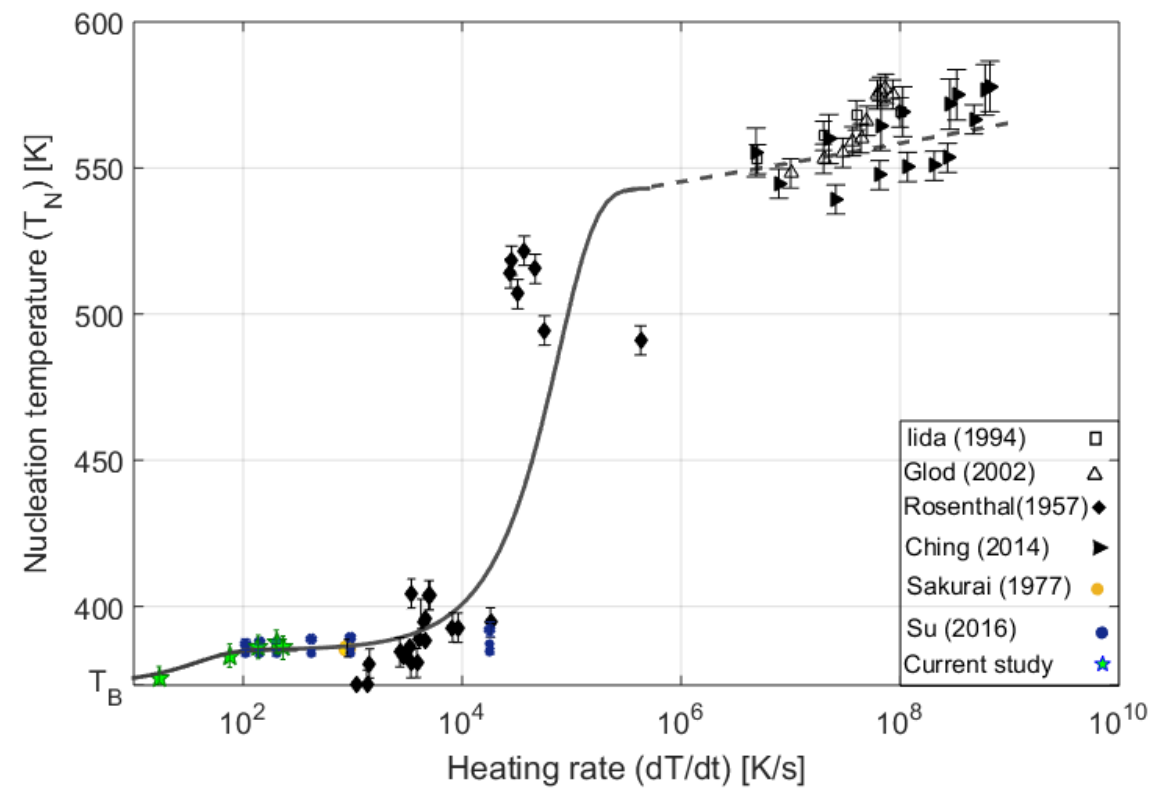

Figure 5: Nucleation temperature vs. heating rate - a comparison between experimentally observed values (see the insert) and those inferred from Equations (19) (solid curve) and (18) (dashed curve). The experimental points referred to as Iida (1994), Glod (2002), Rosenthal (1957), Ching (2014), Sakurai (1977), and Su (2016) were taken from [23, 24, 25, $26,27,13]$, respectively. The points referred to as Current study are original experimental results.

Our experimentally observed values of the degree of superheating $\left(\Delta T_{S H}=\right.$ $T_{N}-T_{B}$ ), those presented by $\mathrm{Su}$ et al. [13], and those predicted by Eq. (20) are shown in Figure 6. Good agreement between our experimental results, those reported in [13] and predicted by (20) can be clearly seen from this figure. The observed values of $\Delta T_{S H}$ (up to about $15 \mathrm{~K}$ ) should be taken into account when modelling the puffing/micro-explosion phenomena.

Error bars are shown on both Figures 5 and 6 . Average errors of temperature measurements reported in $[23,24,25]$ were $\pm 5 \mathrm{~K}$; those reported in [13] were $\pm 1.5 \%$; those observed in the original experiments were $\pm 4 \mathrm{~K}$. The authors of [27] did not describe the errors of their measurements.

It would not be easy for us to develop a single correlation for $T_{N}$ instead of the above-mentioned three correlations as the physical nature of the onset of nucleate boiling (ONB) is expected to be different for different heating 


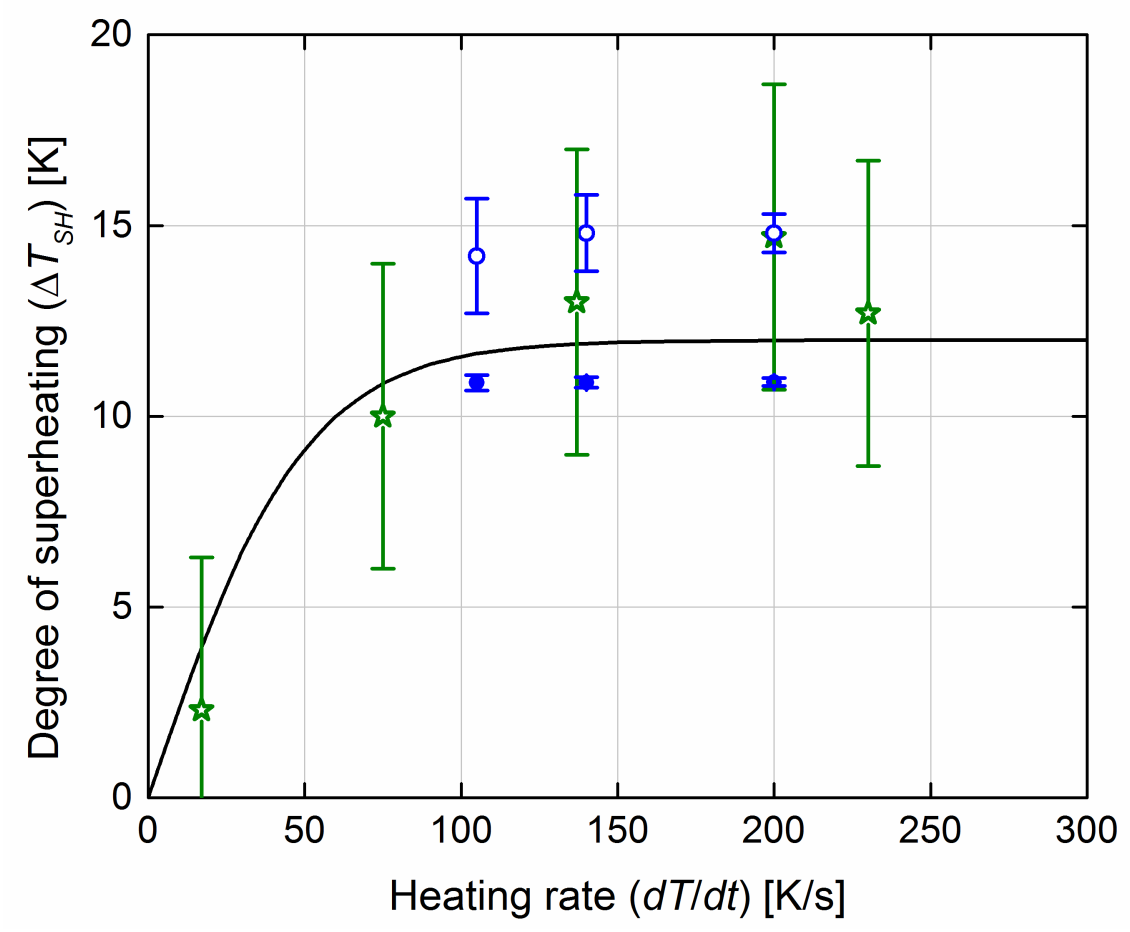

Figure 6: Superheating values of $\Delta T_{S H}=T_{N}-T_{B}$ observed in our experiments (stars), the experiments by Su et al. at different initial subcooling [13] (filled and empty circles) and those inferred from Eq. (20) (solid curve) for the values of input parameters described in the text.

rates, as discussed earlier in this section.

\section{Times to puffing/micro-explosion}

The analysis presented in the previous section allows us to revise the times to puffing obtained in Section 3 assuming that the puffing starts when the temperature at the water/fuel interface reaches the boiling temperature of water. This revision is based on the observation that the model described in Section 3 allows us not only to obtain the values of the temperature at the water/fuel interface, but also the rate of increase of this temperature at this location $(\dot{T})$. Knowledge of $\dot{T}$ allows us to obtain the values of the nucleation temperature at the fuel vapour interface at each time instant using one of the correlations suggested in Section 4. This in turn allows us to obtain a 
more accurate time to puffing/micro-explosion as the time instant when the interface temperature is equal to the nucleation temperature.

In what follows this new approach to estimation of the time to puffing/ micro-explosion, using the results presented in Section 4, is illustrated for the cases shown in Figures 2-4.

The plots of $\dot{T}$ at the water/fuel interface versus time for the same input parameters as in Figure 2 are shown in Figure 7. As can be seen from this figure, for almost the whole time period under consideration the values of $\dot{T}$ are in the range $10^{2} \leq \dot{T} \leq 10^{6} \mathrm{~K} / \mathrm{s}$ which allowed us to use Approximation (19) to estimate $T_{N}$.

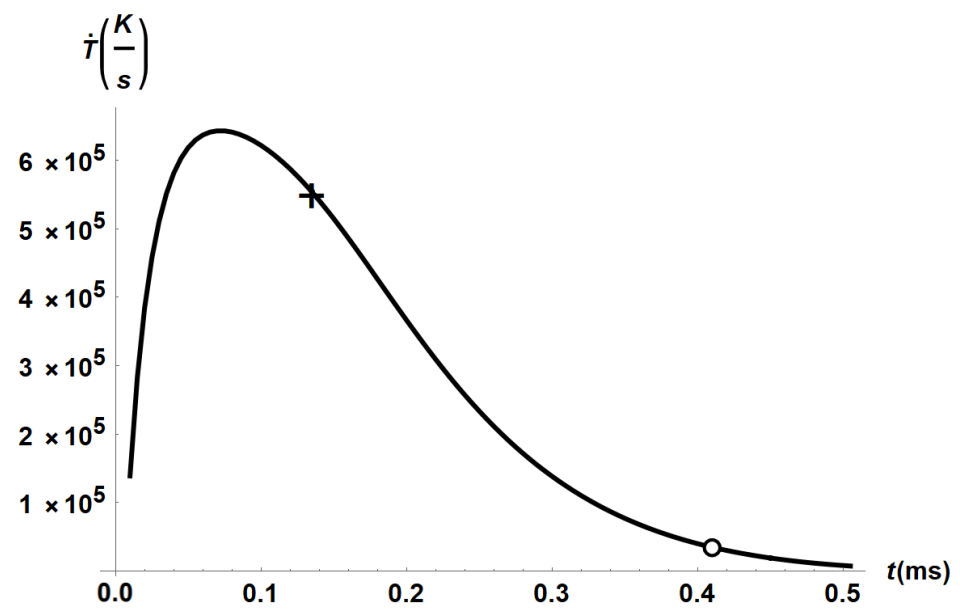

Figure 7: The plot of $\dot{T} \equiv d T / d t$ at the water/fuel interface versus time, for the values of parameters used in Figure 2; the cross and circle indicate the time instant when $T_{w}=T_{B}$ and $T_{w}=T_{N}$, respectively (see Fig. 8).

The plots of the nucleation temperature $\left(T_{N}\right)$, inferred from Correlation (18) and the values of $\dot{T}$ shown in Figure 7, versus time, are shown in Figure 8. In the same figure, the plots of the droplet surface temperature $\left(T_{s}\right)$ and the temperature at the water/fuel interface $\left(T_{w}\right)$ versus time are shown alongside the line corresponding to the boiling temperature of water $\left(T_{B}\right)$.

It follows from Figure 8 that the curves $T_{N}$ versus time and $T_{w}$ versus time intersect at $0.41 \mathrm{~ms}$. As in the previous cases, this time can be associated with the time to puffing/micro-explosion in the new model where the time instant when $T_{w}=T_{N}$ is associated with the start of puffing/micro-explosion. This time can be compared with the time to puffing/micro-explosion equal 


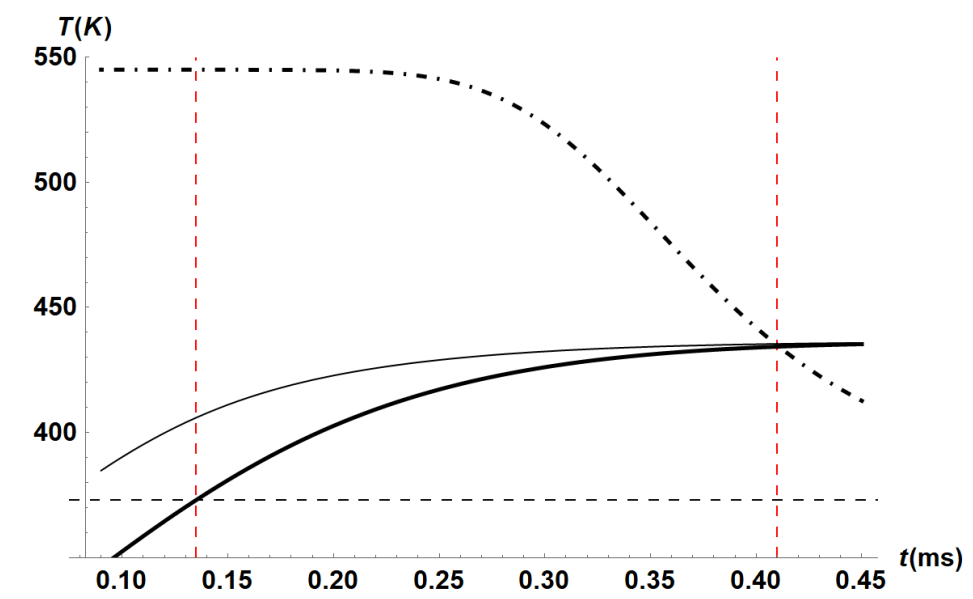

Figure 8: The plots of time evolution of $T_{w}$ (thick solid), $T_{s}$ (thin solid), $T_{N}$ (dasheddotted), and $T_{B}$ (horizontal dashed line); the vertical dashed lines show the time instant when $T_{w}=T_{B}$ and $T_{w}=T_{N}$; the calculations were performed for the same values of parameters as in Figure 2.

to $0.135 \mathrm{~ms}$ predicted by the model described in Section 3, where the time to puffing/micro-explosion was associated with the time instant when $T_{w}=T_{B}$. The difference between these times certainly needs to be taken into account when modelling puffing/micro-explosion phenomena.

Note that at $t=0.41 \mathrm{~ms}$ the droplet radius reduces to $3.86 \mu \mathrm{m}$ which is well above the radius of the water sub-droplet $(2.66 \mu \mathrm{m})$ for $V_{w 0}=15 \%$.

The plots of $\dot{T}$ at the water/fuel interface versus time for the same input parameters as in Figures 3 and 4 (large droplet heating and evaporating with and without taking into account the effect of swelling) are shown in Figure 9. As can be seen from this figure, the values of $\dot{T}$ are smaller than $11 \mathrm{~K} / \mathrm{s}$ in this case. This allows us to use Correlation (20) for the estimation of the nucleation temperature. Note that the maximal values of $\dot{T}$ are slightly smaller for the case with swelling than for the case without swelling.

The plots of the nucleation temperature, inferred from Correlation (20) and the values of $\dot{T}$ shown in Figure 9 for the case without swelling, versus time are shown in Figure 10. In the same figure, the plots for the droplet surface temperature $\left(T_{s}\right)$, the temperature at the water/fuel interface $\left(T_{w}\right)$ and the line corresponding to the boiling temperature of water $\left(T_{B}\right)$ are shown.

It follows from Figure 10 that, in contrast to the case shown in Figure 


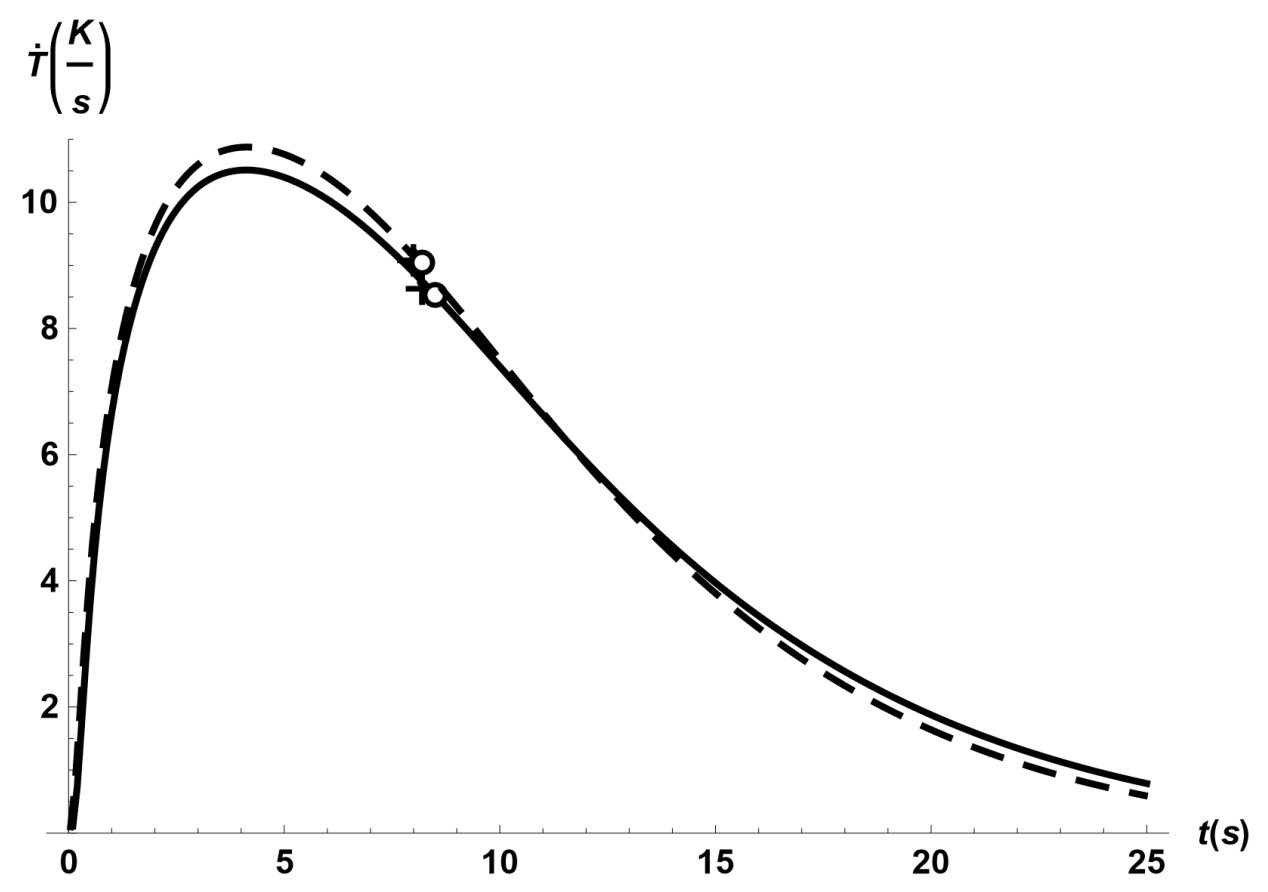

Figure 9: The plot of $\dot{T}$ at the water/fuel interface versus time for the values of parameters used in Figures 3 and 4, taking into account the effects of swelling (solid) and ignoring them (dashed); the crosses and circles indicate the time instants when $T_{w}=T_{B}$ and $T_{w}=T_{N}$, respectively (see Figs. 10 and 11 for the details).

8 , the curves $T_{N}$ versus time and $T_{w}$ versus time intersect at $8.2 \mathrm{~s}$. This time can be associated with the time to puffing/micro-explosion in the new model where the time instant when $T_{w}=T_{N}$ is associated with the start of puffing/micro-explosion. This time can be compared with the time to puffing/micro-explosion equal to $8.0 \mathrm{~s}$ predicted by the model described in Section 3, where the time to puffing/micro-explosion was associated with the time instant when $T_{w}=T_{B}$ (also shown in Figure 10).

Plots similar to those shown in Figure 10 but taking into account the effect of swelling are shown in Figure 11. The shapes of the curves shown in Figures 11 and 10 are rather similar, although in Figures 11 the time to puffing/microexplosion predicted by the new model with swelling $(8.5 \mathrm{~s})$ is slightly longer than the one predicted by the model not taking into account the effect of swelling. This value can be considered the most accurate one within the restrictions of our model. It is slightly larger than the one predicted by the 


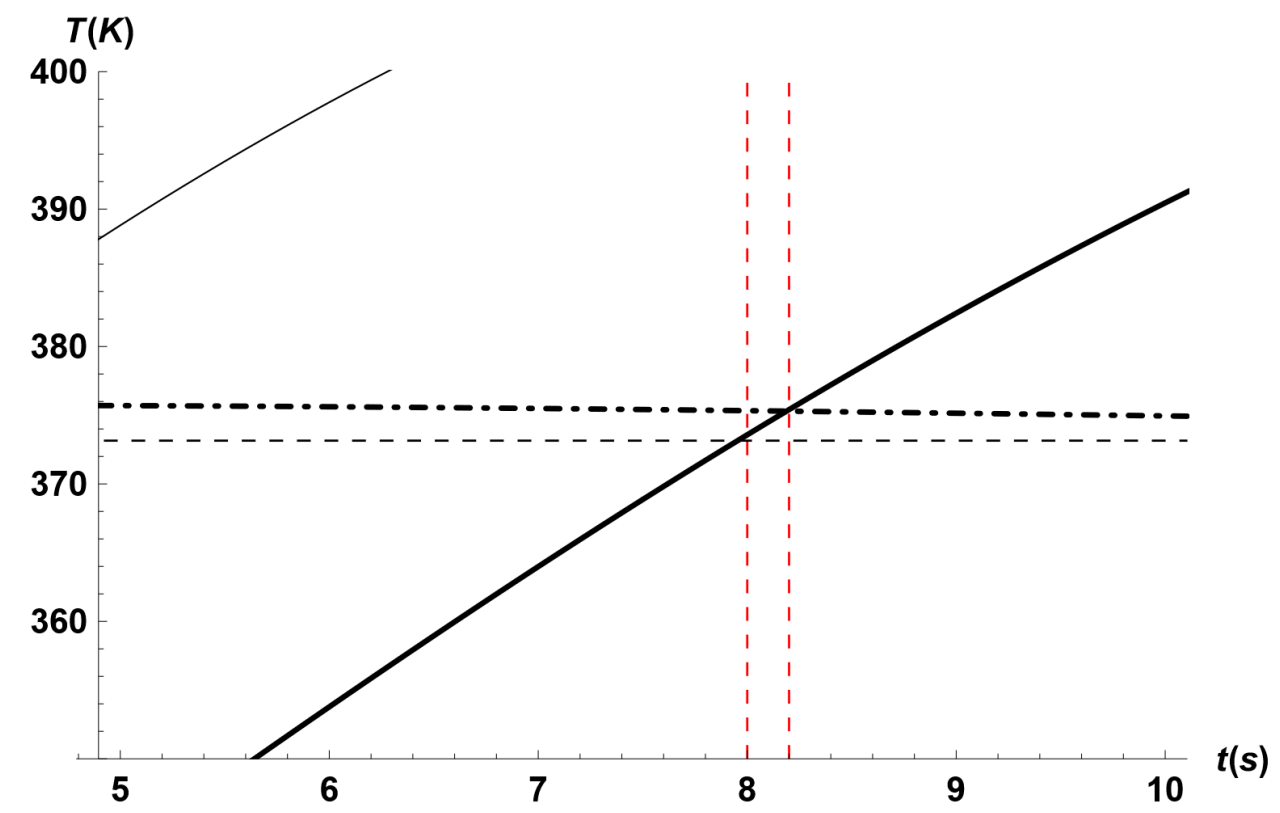

Figure 10: The same as Figure 8 but for the values of parameters and approximations used in Figure 3; vertical dashed lines show time instants when $T_{w}=T_{B}$ and $T_{w}=T_{N}$.

model described in Section 3, where the time to puffing/micro-explosion was associated with the time instant when $T_{w}=T_{B}(8.2 \mathrm{~s})$.

Note that at $t=8.2 \mathrm{~s}$ the droplet radius reduces, due to the combined effect of swelling and evaporation, to $1.31 \mathrm{~mm}$ which is well above the radius of the water sub-droplet $(0.78 \mathrm{~mm})$ for $V_{w 0}=20 \%$.

As follows from our previous analysis in this section, our model predicts a very strong effect of $T_{N}$ on the onset of puffing/micro-explosion for very small droplets (see Figure 8) and relatively weak effect of $T_{N}$ on the time to puffing/micro-explosion for large droplets (see Figures 10 and 11). At the final stage of our analysis we will apply the model described earlier in the paper to investigate the effects of the difference between $T_{N}$ and $T_{B}$ on the time to puffing for intermediate droplets.

Following [11], we assume that the initial n-dodecane droplet radius and temperature are equal to $100 \mu \mathrm{m}$ and $300 \mathrm{~K}$, respectively. Gas temperature is taken as $700 \mathrm{~K}$. The ambient pressure is set to $101.325 \mathrm{kPa}$ and the initial volume fraction of water $V_{w 0}$ is taken to be $15 \%$. The temperature dependence of thermodynamic and transport properties is assumed to be the same 


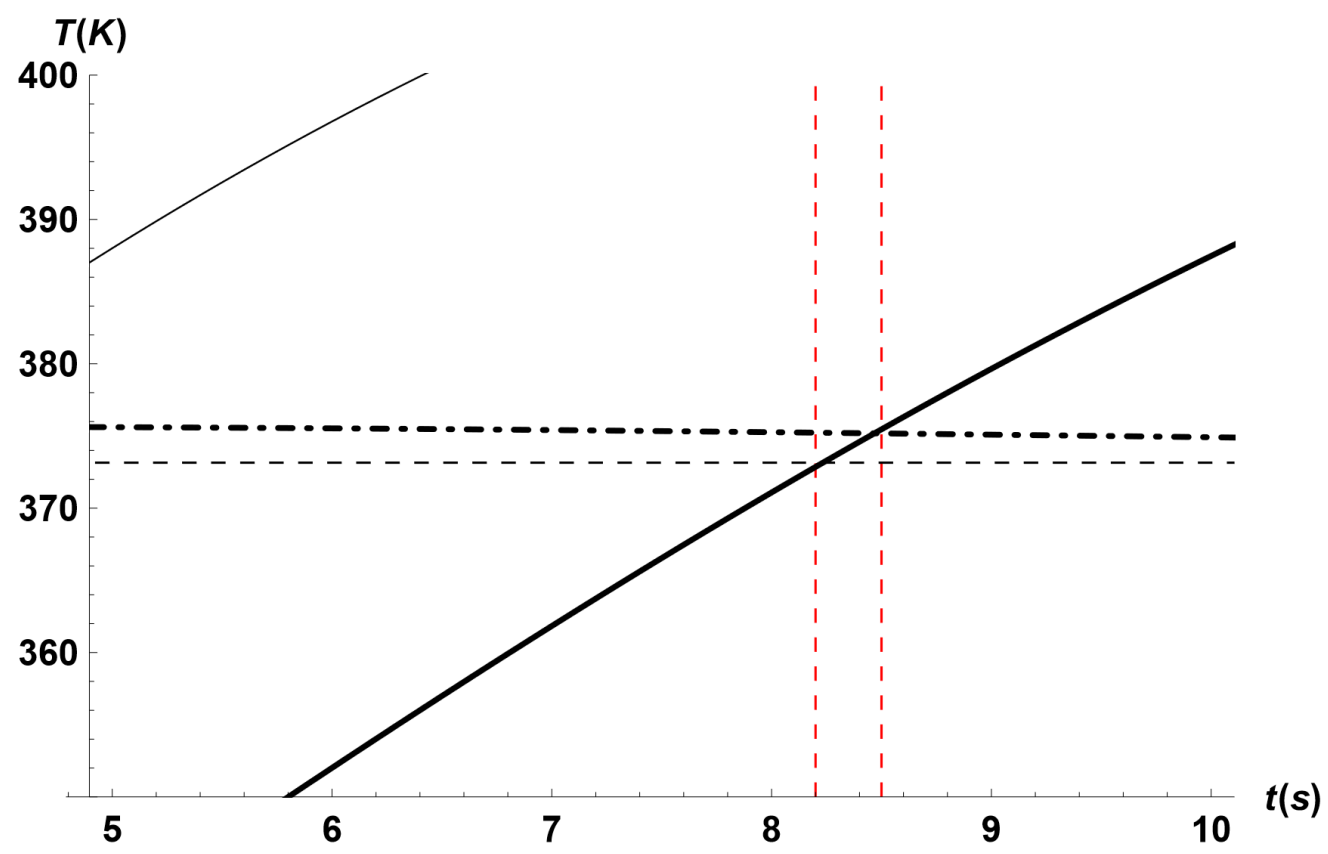

Figure 11: The same as Figure 10 but taking into account the effects of thermal swelling.

as for the previous figures.

These input parameters allowed us to prepare plots similar to those shown in Figures 2, 3 and 4 but for the case of intermediate droplets. The results of calculations allowed us to prepare the plot of $\dot{T}$ at the water/fuel interface versus time, taking into account the effect of swelling. This plot is shown in Figure 12. The cross and circle in this figure show the time instants when the temperature at the water/fuel interface reaches the water boiling and nucleation temperatures, respectively. In both cases, $\dot{T}$ was in the range $10^{2} \leq \dot{T} \leq 10^{6} \mathrm{~K} / \mathrm{s}$, which allowed us to use Approximation (19) to estimate $T_{N}$.

The plots of the nucleation temperature, inferred from Correlation (19) and the values of $\dot{T}$ shown in Figure 12, are shown in Figure 13. In the same figure, the plots for the droplet surface temperature $\left(T_{s}\right)$, the temperature at the water/fuel interface $\left(T_{w}\right)$ and the line corresponding to the boiling temperature of water $\left(T_{B}\right)$ are shown, as in the case of Figures 8,10 and 11 .

It follows from Figure 13 that the curves $T_{N}$ versus time and $T_{w}$ versus time intersect at $67 \mathrm{~ms}$. As in the previous cases, this time can be associated 


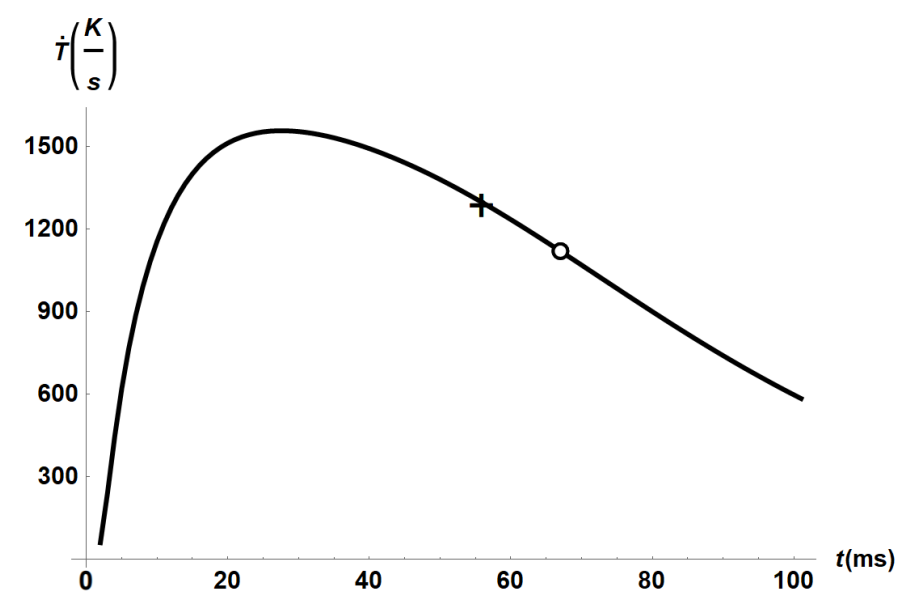

Figure 12: The plot of $\dot{T} \equiv d T / d t$ at the water/fuel interface versus time, for a droplet with initial radius $100 \mu \mathrm{m}$; the cross and circle indicate the time instant when $T_{w}=T_{B}$ and $T_{w}=T_{N}$, respectively (see Figure 13 for the details).

with the time to puffing/micro-explosion in the new model where the time instant when $T_{w}=T_{N}$ is associated with the start of puffing/micro-explosion. This time can be compared with the time to puffing/micro-explosion equal to $56 \mathrm{~ms}$ predicted by the model described in Section 3, where the time to puffing/micro-explosion was associated with the time instant when $T_{w}=T_{B}$. The difference between these times certainly needs to be taken into account when modelling puffing/micro-explosion phenomena.

Note that at $t=67 \mathrm{~ms}$ the droplet radius increases, due to the combined effect of swelling and evaporation, to $101.65 \mu \mathrm{m}$ which is well above the radius of the water sub-droplet $(53.13 \mu \mathrm{m})$ for $V_{w 0}=15 \%$.

The experimentally observed value of the time to puffing/micro-explosion, for large droplets and the same parameters as used for Figure 11, was 3.075 \pm $0.2 \mathrm{~s}$. Similar values for droplets with initial radius $100 \mu \mathrm{m}$ and the same parameters as used for Figures 12 and 13, were less than $12 \mathrm{~ms}[1,11]$. In both cases the predicted values of the time to puffing/micro-explosion are noticeably longer compared with those observed experimentally. As in the case considered in [2], this difference is related to the key assumption of our model, that a water sub-droplet is located exactly in the centre of the fuel droplet. This assumption is questionable in the case of the intermediate and relatively large droplets considered in our experiment and in $[1,11]$. We are not aware of accurate measurements of time to puffing for small droplets with 


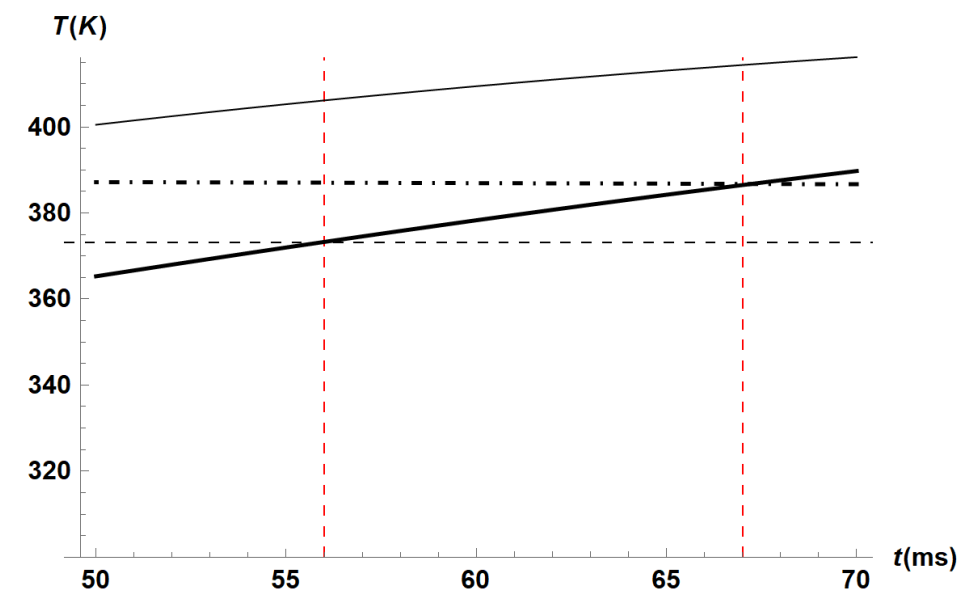

Figure 13: The plots of time evolution of $T_{w}$ (thick solid), $T_{s}$ (thin solid), $T_{N}$ (dasheddotted), and $T_{B}$ (horizontal dashed line); the vertical dashed line shows the time instant when $T_{w}=T_{B}$; the calculations were performed for a droplet with initial radius $100 \mu \mathrm{m}$.

radii less than $5 \mu \mathrm{m}$.

As follows from our analysis, in all cases, the difference in the time to puffing/micro-explosion, inferred from the assumptions that puffing starts at water boiling and nucleation temperatures, needs to be taken into account if accurate estimation of this time is required, regardless of which model for puffing/micro-explosion is used in the analysis.

\section{Conclusions}

A new approach to modelling puffing and micro-explosions in composite water/fuel droplets is suggested and discussed. This approach is based on the same assumption as used in the models described in $[1,11]$ that a spherical water sub-droplet is located in the centre of a spherical fuel (n-dodecane) droplet. As in [11] the heating of the composite droplet is described by the heat conduction equation with the Robin boundary condition at its surface and the continuity conditions at the fuel-water interface.

The analytical solution to this equation is obtained at each time step and incorporated into the numerical code used for the analysis of droplet heating and evaporation. The Abramzon and Sirignano model is used for the approximation of the droplet evaporation process for arbitrary Lewis numbers and the effect of evaporation on the heating process is taken into account. 
The effects of thermal swelling of droplets are also taken into account.

The results of calculation using this code allowed us to obtain the time evolution of the temperature at the water-fuel interface and the evolution of the time derivative of this temperature $(\dot{T}=d T / d t)$ with time in the same location.

Using the published and original experimental data, two new correlations for the nucleation temperatures $T_{N}$ as functions of $\dot{T}$ are suggested. The first one is valid for $10^{2} \leq \dot{T} \leq 10^{6}$, while the second one is valid for $0 \leq \dot{T} \leq 300$. Using these correlations and the values of $\dot{T}$ predicted by the code, the time evolutions of the nucleation temperatures $T_{N}$ at the water-fuel interfaces are obtained. The predicted values of $T_{N}$ are compared with the values of temperature at this interface $T_{w}$.

The time instant when $T_{w}=T_{N}$ is associated with the start of puffing $/$ microexplosion. This approach is different from the one used in $[1,11]$ where the start of puffing/ micro-explosion was associated with the time instant when $T_{w}$ is equal to the boiling temperature of water $T_{B}$.

For relatively large $\left(R_{d 0} \approx 1.33 \mathrm{~mm}\right)$ and intermediate $\left(R_{d 0}=100 \mu \mathrm{m}\right)$ droplets the predicted times to puffing/micro-explosion were larger than those observed experimentally. This is linked with the main limitation of the model, the assumption that the water sub-droplet is located exactly in the centre of the fuel droplet.

\section{Acknowledgements}

Work on this paper was supported by the EPSRC, UK (Grant nos. EP/M002608/1 and EP/R012024/1), the Royal Society (UK) (Grant no. IEC 192007) (contributions by O. Rybdylova and S.S. Sazhin), the Centre for Automotive Research and Electric Mobility (CAREM) of the Universiti Teknologi PETRONAS (UTP) (supported by the ministry of higher education Fundamental Research Grant Scheme (FRGS) (Grant FRGS/1/2017/TK10/UTP/01/2) and UTP Graduate Assistant (GA) studentship) (contribution by Z. Nissar), the National Research Tomsk Polytechnic University (project VIUISHFVP-60/2019) (contribution by P.A. Strizhak), and a Scholarship from the President of the Russian Federation (MN-2.4/3052) (contribution by D.V. Antonov). 


\section{Appendix 1}

\section{Derivation of Formula (11)}

Introducing a new variable

$$
u=T R
$$

we can simplify Equation (1) and the initial condition to:

$$
\begin{gathered}
\frac{\partial u}{\partial t}=\kappa \frac{\partial^{2} u}{\partial R^{2}}+R P(t, R), \\
\left.u\right|_{t=0}=\left\{\begin{array}{lll}
R T_{w 0}(R) & \text { when } 0 \leq R \leq R_{w} \\
R T_{f 0}(R) & \text { when } \quad R_{w}<R \leq R_{d} .
\end{array}\right.
\end{gathered}
$$

The boundary conditions for Equation (A1) can be presented as:

$$
\begin{gathered}
\left.u\right|_{R=R_{w}^{-}}=\left.u\right|_{R=R_{w}^{+}},\left.k_{w}\left[R_{w} u_{R}^{\prime}-u\right]\right|_{R=R_{w}^{-}}=\left.k_{f}\left[R_{w} u_{R}^{\prime}-u\right]\right|_{R=R_{w}^{+}}, \\
{\left.\left[u_{R}^{\prime}+H u\right]\right|_{R=R_{d}}=\mu}
\end{gathered}
$$

where

$$
H=\frac{h}{k_{f}}-\frac{1}{R_{d}} ; \quad \mu=\frac{R_{d}}{k_{f}}\left(h T_{g}+\rho_{l} L_{f} \dot{R}_{d}\right) .
$$

Recalling the definition of $T_{\text {eff }}$, the expression for $\mu$ can be re-written as $\mu=\frac{R_{d} h}{k_{f}} T_{\text {eff }}$. The same applies to $\mu_{0}$ which is introduced later in Formula (A8).

Having introduced the new variable and functions

$$
\xi=R / R_{d}, \quad \tilde{P}(\xi)=P(R) \text { and } F(t, \xi)=u(t, R)
$$

and assuming that $R_{d}$ is constant during the individual time step except in the expression for $\mu^{2}$, we re-write (A1) as

$$
R_{d}^{2} \frac{\partial F}{\partial t}=\kappa \frac{\partial^{2} F}{\partial \xi^{2}}+R_{d}^{3} \xi \tilde{P}(\xi)
$$

\footnotetext{
${ }^{2}$ We take into account heat of evaporation due to the reduction of droplet radius during the evaporation process, but not the change in shape of the droplet during individual time steps.
} 
where

$$
0 \leq \xi \leq 1
$$

The initial and boundary conditions for Equation (A5) can be presented as:

$$
\left.F\right|_{t=0}= \begin{cases}R_{d} \xi T_{w 0}\left(R_{d} \xi\right) & \text { when } 0 \leq \xi \leq \xi_{w} \\ R_{d} \xi T_{f 0}\left(R_{d} \xi\right) & \text { when } \quad \xi_{w}<\xi \leq 1\end{cases}
$$

$\left(\xi_{w}=R_{w} / R_{d}\right)$

$$
\begin{gathered}
\left.F\right|_{\xi=\xi_{w}^{-}}=\left.F\right|_{\xi=\xi_{w}^{+}},\left.k_{w}\left[\xi_{w} F_{\xi}^{\prime}-F\right]\right|_{\xi=\xi_{w}^{-}}=\left.k_{f}\left[\xi_{w} F_{\xi}^{\prime}-F\right]\right|_{\xi=\xi_{w}^{+}}, \\
{\left.\left[F_{\xi}^{\prime}+h_{0} F\right]\right|_{\xi=1}=\mu_{0},}
\end{gathered}
$$

where

$$
h_{0}=H R_{d}=\frac{h R_{d}}{k_{f}}-1 ; \quad \mu_{0}=\mu R_{d}=\frac{R_{d}^{2}}{k_{f}}\left(h T_{g}+\rho_{l} L_{f} \dot{R}_{d}\right) .
$$

We present the convection heat transfer coefficient $h=k_{g} \mathrm{Nu} /\left(2 R_{d}\right)$, where the Nusselt number for stationary evaporating droplets is presented as:

$$
\mathrm{Nu}=2\left[\ln \left(1+B_{T}\right)\right] / B_{T},
$$

$B_{T}$ is the Spalding heat transfer number ${ }^{3}$.

To reduce the boundary condition (Equation (A8)) into a homogeneous one, new function $V(t, \xi)$ is introduced via the equation:

$$
F(t, \xi)=V(t, \xi)+\frac{\mu_{0}}{1+h_{0}} \xi
$$

Expression (A.10) allows us to re-write Equation (A5) and initial and boundary conditions (A6)-(A8) as

$$
\begin{gathered}
R_{d}^{2} \frac{\partial V}{\partial t}=\kappa \frac{\partial^{2} V}{\partial \xi^{2}}+R_{d}^{3} \xi \tilde{P}(\xi), \\
V(t=0, \xi)=R_{d} \xi T_{0}\left(R_{d} \xi\right)-\frac{\mu_{0}}{1+h_{0}} \xi
\end{gathered}
$$

\footnotetext{
${ }^{3}$ The effect of evaporation on $\mathrm{Nu}$ was ignored in [11] where it was assumed that $\mathrm{Nu}=2$.
} 


$$
\begin{gathered}
\left.T_{0}\left(R_{d} \xi\right)\right|_{t=0}=\left\{\begin{array}{lll}
T_{w 0}\left(R_{d} \xi\right) & \text { when } 0 \leq \xi \leq \xi_{w} \\
T_{f 0}\left(R_{d} \xi\right) & \text { when } \quad \xi_{w}<\xi \leq 1,
\end{array}\right. \\
\left.V\right|_{\xi=\xi_{w}^{-}}=\left.V\right|_{\xi=\xi_{w}^{+}},\left.k_{w}\left[\xi_{w} V_{\xi}^{\prime}-V\right]\right|_{\xi=\xi_{w}^{-}}=\left.k_{f}\left[\xi_{w} V_{\xi}^{\prime}-V\right]\right|_{\xi=\xi_{w}^{+}}, \\
{\left.\left[V_{\xi}^{\prime}+h_{0} V\right]\right|_{\xi=1}=0 .}
\end{gathered}
$$

We look for the following solution to Equation (A11):

$$
V=\sum_{n=1}^{\infty} \Theta_{n}(t) v_{n}(\xi)
$$

where $v_{n}(\xi)$ form the full set of non-trivial solutions to the eigenvalue prob$\mathrm{lem}^{4}$ :

$$
\frac{\mathrm{d}^{2} v}{\mathrm{~d} \xi^{2}}+a^{2} \lambda^{2} v=0
$$

subject to boundary conditions:

$$
\begin{aligned}
& \left.\begin{array}{l}
\left.v\right|_{\xi=0}=\left.\left(v_{\xi}^{\prime}+h_{0} v\right)\right|_{\xi=1}=0 \\
\left.v\right|_{\xi=\xi_{w}^{-}}=\left.v\right|_{\xi=\xi_{w}^{+}} \\
\left.k_{w}\left[\xi_{w} v_{\xi}^{\prime}-v\right]\right|_{\xi=\xi_{w}^{-}}=\left.k_{f}\left[\xi_{w} v_{\xi}^{\prime}-v\right]\right|_{\xi=\xi_{w}^{+}}
\end{array}\right\}, \\
& a=\frac{1}{\sqrt{\kappa}}= \begin{cases}\sqrt{\frac{c_{w} \rho_{w}}{k_{w}}} \equiv a_{w} & \text { when } \quad 0 \leq \xi \leq \xi_{w} \\
\sqrt{\frac{c_{f} \rho_{f}}{k_{f}}} \equiv a_{f} & \text { when } \quad \xi_{w}<\xi \leq 1 .\end{cases}
\end{aligned}
$$

Note that $\lambda$ has dimensions of length $/ \sqrt{\text { time. }}$

The general solution to (A17) is as follows:

$$
v(\xi)=\left\{\begin{array}{lll}
A_{1} \cos \left(\lambda a_{w} \xi\right)+B_{1} \sin \left(\lambda a_{w} \xi\right) & \text { when } \quad 0 \leq \xi \leq \xi_{w} \\
A_{2} \cos \left(\lambda a_{f} \xi\right)+B_{2} \sin \left(\lambda a_{f} \xi\right) & \text { when } \quad \xi_{w}<\xi \leq 1
\end{array}\right.
$$

Note that:

$$
A_{2} \cos \left(\lambda a_{f} \xi\right)+B_{2} \sin \left(\lambda a_{f} \xi\right)=\sqrt{A_{2}^{2}+B_{2}^{2}} \sin \left(\lambda a_{f} \xi+\tan ^{-1}\left(\frac{A_{2}}{B_{2}}\right)\right) .
$$

\footnotetext{
${ }^{4}$ The term $a^{2}$ was not taken into account in Equation (A1.20) of [11]; this led to the situation in which $\Theta_{n}(t)$ depended on $\xi$ via $\kappa$ which is not compatible with the original assumption used when presenting (A16).
} 
To satisfy the first equation in System (A18) we need to assume that $A_{1}=0$. Having introduced new coefficients:

$$
B=B_{1}, \quad A=\sqrt{A_{2}^{2}+B_{2}^{2}} \text { and } \beta=\tan ^{-1}\left(\frac{A_{2}}{B_{2}}\right)
$$

we can rewrite (A20) as:

$$
v(\xi)=\left\{\begin{array}{lll}
B \sin \left(\lambda a_{w} \xi\right) & \text { when } \quad 0 \leq \xi \leq \xi_{w} \\
A \sin \left(\lambda a_{f} \xi+\beta\right) & \text { when } \quad \xi_{w}<\xi \leq 1 .
\end{array}\right.
$$

From the middle equation in System (A18) we obtain:

$$
B \sin \left(\lambda a_{w} \xi_{w}\right)=A \sin \left(\lambda a_{f} \xi_{w}+\beta\right) .
$$

This equation is satisfied when:

$$
B=\frac{1}{\sin \left(\lambda a_{w} \xi_{w}\right)}, \quad \text { and } \quad A=\frac{1}{\sin \left(\lambda a_{f} \xi_{w}+\beta\right)},
$$

$\beta$ is obtained from the flux exchange at the interface given in the last equations in System (A18):

$$
\beta=\cot ^{-1}\left[\frac{k_{f}-k_{w}}{k_{f} a_{f} \xi_{w} \lambda}+\frac{k_{w} a_{w}}{k_{f} a_{f}} \cot \left(a_{w} \lambda \xi_{w}\right)\right]+i \pi-a_{f} \lambda \xi_{w},
$$

where $i=0,1,2,3, \ldots . .{ }^{5}$.

We consider only the case when $i=0$. The values of $v$ would be the same for other $i$.

A countable set of positive eigenvalues $\lambda_{n}$ is obtained from the boundary condition at $\xi=1$ (solution to the first equation in System (A18)):

$$
\lambda_{n} a_{f} \cos \left(\lambda_{n} a_{f}+\beta\right)+h_{0} \sin \left(\lambda_{n} a_{f}+\beta\right)=0 .
$$

The values of $v$ for $\lambda=\lambda_{n}$ are known as eigenfunctions and referred to as $v_{n}$. For homogeneous droplets, when $\beta=0$, Equation (A25) is equivalent to Equation (3.38) of [12] if we formally replace $\lambda_{n} a_{f}$ in (A25) with $\lambda$.

\footnotetext{
${ }^{5}$ For homogeneous droplets, in which properties of water and fuel are the same, $\beta=0$.
} 
The explicit expressions for $v_{n}$ for $\lambda_{n}$ inferred from (A25) follow from (A22) remembering the expressions for $A$ and $B$ :

$$
v_{n}(\xi)= \begin{cases}\frac{\sin \left(\lambda_{n} a_{w} \xi\right)}{\sin \left(\lambda_{n} a_{w} \xi_{w}\right)} & \text { when } 0 \leq \xi \leq \xi_{w} \\ \frac{\sin \left(\lambda_{n} a_{f} \xi+\beta_{n}\right)}{\sin \left(\lambda_{n} a_{f} \xi_{w}+\beta_{n}\right)} & \text { when } \quad \xi_{w}<\xi \leq 1\end{cases}
$$

where $\beta_{n}$ is $\beta\left(\lambda=\lambda_{n}\right)$.

Functions $v_{n}(\xi)$ are orthogonal with weight (see [12])

$$
b=\left\{\begin{array}{ll}
k_{w} a_{w}^{2}=c_{w} \rho_{w} & \text { when } \quad 0 \leq \xi \leq \xi_{w} \\
k_{f} a_{f}^{2}=c_{f} \rho_{f} & \text { when } \quad \xi_{w}<\xi \leq 1
\end{array} .\right.
$$

Thus $\int_{0}^{1} v_{n}(\xi) v_{m}(\xi) b \mathrm{~d} \xi=\delta_{n m}\left\|v_{n}\right\|^{2}$, where

$$
\delta_{n m}=\left\{\begin{array}{lll}
1 & \text { when } & n=m \\
0 & \text { when } & n \neq m
\end{array} .\right.
$$

The completeness of this set of functions is discussed in [12].

The norm of $v_{n}$ with weight $b$ is given by the following expression:

$$
\begin{gathered}
\left\|v_{n}\right\|^{2}=\int_{0}^{1} v_{n}^{2} b \mathrm{~d} \xi \\
=\int_{0}^{\xi_{w}}\left[\frac{\sin \left(\lambda_{n} a_{w} \xi\right)}{\sin \left(\lambda_{n} a_{w} \xi_{w}\right)}\right]^{2} c_{w} \rho_{w} \mathrm{~d} \xi+\int_{\xi_{w}}^{1}\left[\frac{\sin \left(\lambda_{n} a_{f} \xi+\beta_{n}\right)}{\sin \left(\lambda_{n} a_{f} \xi_{w}+\beta_{n}\right)}\right]^{2} c_{f} \rho_{f} \mathrm{~d} \xi \\
=\frac{c_{w} \rho_{w}}{\sin ^{2}\left(\lambda_{n} a_{w} \xi_{w}\right)} \int_{0}^{\xi_{w}} \sin ^{2}\left(\lambda_{n} a_{w} \xi\right) \mathrm{d} \xi+\frac{c_{f} \rho_{f}}{\sin ^{2}\left(\lambda_{n} a_{f} \xi_{w}+\beta_{n}\right)} \int_{\xi_{w}}^{1} \sin ^{2}\left(\lambda_{n} a_{f} \xi+\beta_{n}\right) \mathrm{d} \xi \\
=\frac{\sqrt{c_{w} \rho_{w} k_{w}}}{\lambda_{n} \sin ^{2}\left(\lambda_{n} a_{w} \xi_{w}\right)}\left[\frac{a_{w} \lambda_{n} \xi_{w}}{2}-\frac{\sin \left(2 a_{w} \lambda_{n} \xi_{w}\right)}{4}\right] \\
\left.+\frac{a_{f} \lambda_{n}\left(1-\xi_{w}\right)}{2}-\frac{\sin \left(2 \lambda_{n} a_{f}+2 \beta_{n}\right)-\sin \left(2 \lambda_{n} a_{f} \xi_{w}+2 \beta_{n}\right)}{4}\right\} .
\end{gathered}
$$

The fact that functions $v_{n}$ are orthogonal with weight $b$ allows us to perform the following expansions:

$$
f(\xi) \equiv \frac{-\xi}{1+h_{0}}=\sum_{n=1}^{\infty} f_{n}(t) v_{n}(\xi),
$$




$$
\begin{aligned}
& F_{0}(\xi) \equiv R_{d} \xi T_{0}\left(R_{d} \xi\right)=\sum_{n=1}^{\infty} q_{n}(t) v_{n}(\xi) \\
& R_{d}^{3} \xi \tilde{P}(\xi)=\sum_{n=1}^{\infty} p_{n}(t) v_{n}(\xi) \\
& f_{n}=\frac{1}{\left\|v_{n}\right\|^{2}} \int_{0}^{1} f(\xi) v_{n}(\xi) b \mathrm{~d} \xi \\
& =\frac{1}{\left\|v_{n}\right\|^{2}}\left\{\int_{0}^{\xi_{w}} \frac{-\xi}{1+h_{0}} \frac{\sin \left(\lambda_{n} a_{w} \xi\right)}{\sin \left(\lambda_{n} a_{w} \xi_{w}\right)} k_{w} a_{w}^{2} \mathrm{~d} \xi+\int_{\xi_{w}}^{1} \frac{-\xi}{1+h_{0}} \frac{\sin \left(\lambda_{n} a_{f} \xi+\beta_{n}\right)}{\sin \left(\lambda_{n} a_{f} \xi_{w}+\beta_{n}\right)} k_{f} a_{f}^{2} \mathrm{~d} \xi\right\} \\
& q_{n}=\frac{1}{\left\|v_{n}\right\|^{2}} \int_{0}^{1} F_{0}(\xi) v_{n}(\xi) b \mathrm{~d} \xi \\
& =\frac{1}{\left\|v_{n}\right\|^{2}}\left\{\int_{0}^{\xi_{w}} R_{d} \xi T_{0}\left(R_{d} \xi\right) \frac{\sin \left(\lambda_{n} a_{w} \xi\right)}{\sin \left(\lambda_{n} a_{w} \xi_{w}\right)} k_{w} a_{w}^{2} \mathrm{~d} \xi+\int_{\xi_{w}}^{1} R_{d} \xi T_{0}\left(R_{d} \xi\right) \frac{\sin \left(\lambda_{n} a_{f} \xi+\beta_{n}\right)}{\sin \left(\lambda_{n} a_{f} \xi_{w}+\beta_{n}\right)} k_{f} a_{f}^{2} \mathrm{~d} \xi\right\} \\
& p_{n}=\frac{1}{\left\|v_{n}\right\|^{2}} \int_{0}^{1} R_{d}^{3} \xi \tilde{P}(\xi) v_{n}(\xi) b \mathrm{~d} \xi \\
& =\frac{1}{\left\|v_{n}\right\|^{2}}\left\{\int_{0}^{\xi_{w}} R_{d}^{3} \xi \tilde{P}(\xi) \frac{\sin \left(\lambda_{n} a_{w} \xi\right)}{\sin \left(\lambda_{n} a_{w} \xi_{w}\right)} k_{w} a_{w}^{2} \mathrm{~d} \xi+\int_{\xi_{w}}^{1} R_{d}^{3} \xi \tilde{P}(\xi) \frac{\sin \left(\lambda_{n} a_{f} \xi+\beta_{n}\right)}{\sin \left(\lambda_{n} a_{f} \xi_{w}+\beta_{n}\right)} k_{f} a_{f}^{2} \mathrm{~d} \xi\right\} .
\end{aligned}
$$

Having substituted (A16) into (A11) and remembering (A17) we obtain:

$$
\sum_{n=1}^{\infty}\left[R_{d}^{2} \frac{\mathrm{d} \Theta}{\mathrm{d} t}+\lambda_{n}^{2} \Theta\right] v_{n}(\xi)=\sum_{n=1}^{\infty} p_{n} v_{n}(\xi) .
$$

This equation is satisfied if and only if the coefficients on the two sides are equal, which implies that

$$
R_{d}^{2} \frac{\mathrm{d} \Theta}{\mathrm{d} t}+\lambda_{n}^{2} \Theta=p_{n} .
$$

Equation (A35) is solved for the set of countable values of $\lambda$, leading to discrete values of $\Theta\left(\Theta_{n}\right)$ subject to the initial condition:

$$
\Theta_{n}(t=0)=q_{n}+f_{n} \mu_{0} .
$$

The condition (A36) follows from (A12), (A16), (A29) and (A30). 
Assuming that $p_{n}$ is constant during the time step, the solution to this equation is straightforward:

$$
\Theta_{n}(t)=\left(q_{n}+f_{n} \mu_{0}\right) \exp \left(-\frac{\lambda_{n}^{2} t}{R_{d}^{2}}\right)+\frac{p_{n}}{\lambda_{n}^{2}}\left[1-\exp \left(-\frac{\lambda_{n}^{2} t}{R_{d}^{2}}\right)\right] .
$$

Remembering the definitions of $u, F$ and $V$ we obtain the final expression:

$$
T=\frac{1}{R_{d} \xi}\left[\sum_{n=1}^{\infty} \Theta_{n}(t) v_{n}(\xi)+\frac{\mu_{0} \xi}{1+h_{0}}\right]
$$

This expression is the same as (11).

\section{Appendix 2}

\section{Comparison of Equation (A37) with Equation (16) of [17] in the case of homogeneous droplets}

To compare the predictions of the new model and the model developed in [17], we assume that the properties of water and fuel are the same:

$$
k_{w}=k_{f} ; \quad \kappa_{w}=\kappa_{f} ; \quad a_{w}=a_{f}
$$

In what follows we assume that all properties are those of fuel.

Note that

$$
\frac{\mu_{0} \xi}{R_{d}\left(1+h_{0}\right)}=T_{g}+\frac{\rho_{l} L_{f} \dot{R}_{d}}{h} \equiv T_{\mathrm{eff}},
$$

where $T_{\text {eff }}$ is the effective temperature used in Equation (16) of [17].

Having substituted (B1) into (A24) we obtain that $\beta=0$ and the expressions for the eigenfunctions $v_{n}$ (see Expression (A26)) are simplified to:

$$
v_{n}(\xi)=\frac{\sin \left(\tilde{\lambda}_{n} \xi\right)}{\sin \left(\tilde{\lambda}_{n} \xi_{w}\right)} \quad \text { when } \quad 0 \leq \xi \leq 1
$$

where $\tilde{\lambda}_{n}=a_{f} \lambda_{n}$. Remembering that $\beta=0$, Equation (A25) for eigenvalues can be presented as:

$$
\tilde{\lambda}_{n} \cos \tilde{\lambda}_{n}+h_{0} \sin \tilde{\lambda}_{n}=0 .
$$

This equation is identical to Equation (A.3) of [17]. 
Having substituted (B3) into (A27) we obtain:

$$
\begin{gathered}
\left\|v_{n}\right\|^{2}=\int_{0}^{1} v_{n}^{2} c_{f} \rho_{f} \mathrm{~d} \xi \\
=\frac{c_{f} \rho_{f}}{\sin ^{2}\left(\tilde{\lambda}_{n} \xi_{w}\right)} \int_{0}^{1} \sin ^{2}\left(\tilde{\lambda}_{n} \xi\right) \mathrm{d} \xi=\frac{\left.c_{f} \rho_{f}\left\|v_{n}\right\|^{2}\right|_{2004}}{\sin ^{2}\left(\tilde{\lambda}_{n} \xi_{w}\right)}
\end{gathered}
$$

where $\left.\left\|v_{n}\right\|^{2}\right|_{2004}$ is the value of $\left\|v_{n}\right\|^{2}$ used in [17].

Having substituted (B3) into (A31)-(A33) we obtain, remembering (B5):

$$
\begin{gathered}
f_{n}=\frac{1}{\left\|v_{n}\right\|^{2}} \int_{0}^{1} \frac{-\xi}{1+h_{0}} v_{n}(\xi) b \mathrm{~d} \xi \\
=\frac{\sin ^{2}\left(\tilde{\lambda}_{n} \xi_{w}\right)}{\left.c_{f} \rho_{f}\left\|v_{n}\right\|^{2}\right|_{2004}} \frac{c_{f} \rho_{f}}{\sin \left(\tilde{\lambda}_{n} \xi_{w}\right)} \int_{0}^{1} \frac{-\xi}{1+h_{0}} \sin \left(\tilde{\lambda}_{n} \xi\right) \mathrm{d} \xi \\
=\left.\sin \left(\tilde{\lambda}_{n} \xi_{w}\right) f_{n}\right|_{2004}, \\
q_{n}=\frac{1}{\left\|v_{n}\right\|^{2}} \int_{0}^{1} R_{d} \xi T_{0}\left(R_{d} \xi\right) v_{n}(\xi) b \mathrm{~d} \xi \\
=\left.R_{d} \sin \left(\tilde{\lambda}_{n} \xi_{w}\right) q_{n}\right|_{2004}, \\
p_{n}=\frac{1}{\left\|v_{n}\right\|^{2}} \int_{0}^{1} R_{d}^{3} \xi \tilde{P}(\xi) v_{n}(\xi) b \mathrm{~d} \xi \\
=\left.R_{d}^{3} \sin \left(\tilde{\lambda}_{n} \xi_{w}\right) p_{n}\right|_{2004} .
\end{gathered}
$$

Having substituted (B6)-(B8) into (A37) we obtain:

$$
\begin{gathered}
\Theta_{n}(t)=R_{d} \sin \left(\tilde{\lambda}_{n} \xi_{w}\right)\left[\left(\left.q_{n}\right|_{2004}+\left.\mu_{0} f_{n}\right|_{2004}\right) \exp \left(-\frac{\kappa_{f} \tilde{\lambda}_{n}^{2} t}{R_{d}^{2}}\right)\right. \\
\left.+R_{d}^{2} \frac{\left.p_{n}\right|_{2004}}{\kappa_{f} \tilde{\lambda}_{n}^{2}}\left(1-\exp \left(-\frac{\kappa_{f} \tilde{\lambda}_{n}^{2} t}{R_{d}^{2}}\right)\right)\right] .
\end{gathered}
$$

Having substituted (B9) and (B3) into (A38) we obtain Equation (16) of [17], assuming that $\mu_{0}$ is constant during the time step and remembering that $\kappa$, introduced in [17], is equal to $\kappa$ used in our paper divided by $R_{d}^{2}$. 


\section{References}

[1] Sazhin, S.S., Rybdylova, O., Crua, C., Heikal, M., Ismael, M.A., Nissar, Z., Aziz, A.R.B.A. A simple model for puffing/micro-explosions in waterfuel emulsion droplets, International J of Heat and Mass Transfer, 131 (2019) 815-821.

[2] Antonov, D.V., Kuznetsov, G.V., Strizhak, P.A., Rybdylova, O., Sazhin, S.S. Micro-explosion and autoignition of composite fuel/water droplets, Combustion and Flame, 210 (2019) 479-489.

[3] Ogunkoya, D., Li, S., Rojas, O.J., Fang, T. Performance, combustion, and emissions in a diesel engine operated with fuel-in-water emulsions based on lignin, Appl. Energy 154 (2015) 851861.

[4] Taghavifar, H., Anvari, S., Parvishi, A. Benchmarking of water injection in a hydrogen-fueled diesel engine to reduce emissions, Int. J. Hydrogen Energy 42 (16) (2017) 11962-11975

[5] Antonov, D.V., Piskunov, M.V., Strizhak, P.A. Breakup and explosion of droplets of two immiscible fluids and emulsions, Int. J. Thermal Science 142 (2019) 30-41.

[6] Fostiropoulosa, S., Strotos, G., Nikolopoulos, N., Gavaises, M. Numerical investigation of heavy fuel oil droplet breakup enhancement with water emulsions, Fuel, 278 (2020) 118381.

[7] Shinjo, J., Xia, J., Ganippa, L.C., Megaritis, A., Physics of puffing and microexplosion of emulsion fuel droplets. Phys Fluids 25 (2014) 103302. https://doi.org/10.1063/1.4897918.

[8] Shinjo, J., Xia, J., Megaritis, A., Ganippa, L.C., Cracknell, R.F., Modeling temperature distribution inside a emulsion fuel droplet under convective heating: A key to predicting microexplosion and puffing, Atomization and Sprays 26 (2016) 551-583.

[9] Shinjo, J., Xia, J., Combustion characteristics of a single decane/ethanol emulsion droplet and a droplet group under puffing conditions, Proceedings of the Combustion Institute 36 (2017) 2513-2521.

[10] Girin, O.G., Dynamics of the emulsified fuel droplet micro-explosion, Atomization and Sprays 27 (2017) 407-422. 
[11] Nissar, Z., Rybdylova, O., Sazhin, S.S., Heikal, M., Aziz, A.R.B.A., Ismael, M.A., A model for puffing/microexplosions in water/fuel emulsion droplets, International J of Heat and Mass Transfer 149 (2020) 119208.

[12] Sazhin, S.S., Droplets and Sprays, Springer, 2014.

[13] Su, G.-Y., Bucci, M., McKrell, T., Buongiorno, J., Transient boiling of water under exponentially escalating heat inputs. Part I: Pool boiling, International J of Heat and Mass Transfer, 97 (2016) 667-684.

[14] Bar-Kohany, T., Amsalem, Y., Nucleation temperature under various heating rates, International J of Heat and Mass Transfer, 126 (2018) 411-415.

[15] Kadota, T., Yamasaki, H., Recent advances in the combustion of water fuel emulsion, Progress in Energy and Combustion Science 28(5) (2002) 385-404.

[16] Antonov, D., Piskunov, M., Strizhak, P., Tarlet, D., Bellettre, J., Dispersed phase structure and micro-explosion behavior under different schemes of water-fuel droplets heating, Fuel 259 (2020) 116241.

[17] Sazhin, S.S., Krutitskii, P.A., Abdelghaffar, W.A., Sazhina, E.M., Mikhalovsky, S.V., Meikle, S.T., Heikal, M.R., Transient heating of diesel fuel droplets, International J of Heat Mass Transfer 47 (2004) 3327-3340.

[18] Sazhin, S.S., Krutitskii, P.A., Gusev, I.G., Heikal, M., Transient heating of an evaporating droplet, International $\mathrm{J}$ of Heat and Mass Transfer 53(13-14) (2010) 2826-2836.

[19] Yaws, C.L. 'Chapter 3 - Properties Water,' in The Yaws Handbook of Physical Properties for Hydrocarbons and Chemicals (Second Edition), Boston: Gulf Professional Publishing, pp. 811-814 (2015).

[20] Abramzon, B., Sazhin, S.S., Convective vaporization of fuel droplets with thermal radiation absorption, Fuel 85(1) (2006) 32-46.

[21] Skripov, V.P., Metastable Liquids. New York: Wiley; 1974. 
[22] Mikami, M., Kojima, N., An experimental and modeling study on stochastic aspects of microexplosion of binary-fuel droplets, Proceedings of the Combustion Institute 29(1) (2002) 551-559.

[23] Iida, Y., Okuyama, K., Sakurai, K., Boiling nucleation on a very small film heater subjected to extremely rapid heating, International J of Heat and Mass Transfer, 37 (1884) 2771-2780.

[24] Glod, S., Poulikakos, D., Zhao, Z., Yadigaroglu, G., An investigation of microscale explosive vaporization of water on an ultrathin Pt wire, International J of Heat and Mass Transfer, 45 (2002) 367-379.

[25] Rosenthal, M.W., An experimental study of transient boiling, Nuclear Science and Engineering, 2(5) (1957) 640-656.

[26] Ching, E.J., Avedisian, C.T., Carrier, M.J., Cavicchi, R.C., Young, J.R., Land, B.R., Measurement of the bubble nucleation temperature of water on a pulse-heated thin platinum film supported by a membrane using a low-noise bridge circuit, International J of Heat and Mass Transfer, 79 (2014) 82-93.

[27] Sakurai, A., Shiotsu, M., Transient pool boiling heat transfer. I - Incipient boiling superheat, ASME Transactions Journal of Heat Transfer, November 1977. 